\title{
Quantum Radiation Properties of General Nonstationary Black Hole
}

\author{
T. Ibungochouba Singh \\ Department of Mathematics, Manipur University, Canchipur, Manipur 795003, India \\ Correspondence should be addressed to T. Ibungochouba Singh; ibungochouba@rediffmail.com
}

Received 18 July 2016; Revised 30 November 2016; Accepted 4 January 2017; Published 5 March 2017

Academic Editor: Elias C. Vagenas

Copyright (C) 2017 T. Ibungochouba Singh. This is an open access article distributed under the Creative Commons Attribution License, which permits unrestricted use, distribution, and reproduction in any medium, provided the original work is properly cited. The publication of this article was funded by SCOAP ${ }^{3}$.

Using the generalized tortoise coordinate transformations the quantum radiation properties of Klein-Gordon scalar particles, Maxwell's electromagnetic field equations, and Dirac equations are investigated in general nonstationary black hole. The locations of the event horizon and the Hawking temperature depend on both time and angles. A new extra coupling effect is observed in the thermal radiation spectrum of Maxwell's equations and Dirac equations which is absent in the thermal radiation spectrum of scalar particles. We also observe that the chemical potential derived from scalar particles is equal to the highest energy of the negative-energy state of the scalar particle in the nonthermal radiation in general nonstationary black hole. Applying generalized tortoise coordinate transformation a constant term $\xi$ is produced in the expression of thermal radiation in general nonstationary black hole. It indicates that generalized tortoise coordinate transformation is more accurate and reliable in the study of thermal radiation of black hole.

\section{Introduction}

Hawking discovered the thermal radiation of black hole using the techniques of quantum field theory in curve space-time background and the derived radiation spectrum is purely thermal in nature [1,2]. An important aspect in the study of black hole is to reveal the thermal and nonthermal radiation of black hole. References [3-5] have shown that the black hole has a nonzero finite entropy and the entropy of black hole is proportional to the horizon area. References $[6,7]$ also proposed the Hawking radiation as quantum tunneling process where the particles move in the dynamical geometry. They recovered a leading correction to the emission rate arising from the loss mass of the black hole. Following their works, Zhang and Zhao [8-10] have extended the method to more general circumstances for rotating black hole and they have shown the spectrum is no longer precisely thermal. Further, some information of the black hole can be obtained.

Akhmedov et al. [11] investigated the Hawking radiation as tunneling picture in the Schwarzschild black hole using the relativistic Hamilton-Jacobi method. The importance of this investigation is as follows: (i) if Schwarzschild coordinates or any other coordinates related to them via a transformation of spatial coordinates are used, we will get twice original Hawking temperature and (ii) any transformation involving time coordinates is utilized in the black hole evaporation, and it will give the original Hawking result. Following this method, many fruitful results have been obtained in [12-14]. This factor of two issues has been resolved via the discovery of the temporal contribution to the tunneling amplitude in the literatures [15-17]. The thermodynamics of black hole in lovelock gravity and in AdS space-time have been investigated by Cai [18-20]. One of the important aspects in this investigation is to calculate the Hawking temperature.

Recently, Angheben et al. [21] investigated Hawking radiation as tunneling of extremal and rotating black hole using the relativistic Hamilton-Jacobi method and WKB approximation without considering the particles backreaction. Since then many other authors applied the HamiltonJacobi method to study the Hawking radiation for more general space-time [22-25]. Choosing appropriate Gamma matrices, the Hawking radiation as tunneling from Dirac particles was investigated in [26] for the general stationary black hole. By inserting the wave function into the 
Dirac field equations, the action of the radiant particles is derived. This result is related to the Boltzmann factor of emission at the Hawking radiation temperature in accordance with semiclassical WKB approximation. References [27, 28] introduced tortoise coordinate transformation to study the Hawking radiation of black hole in which gravitational field is independent of time. Using tortoise coordinate transformation, Klein-Gordon equation, Maxwell's electromagnetic field equations, and Dirac particles can be transformed into single form of wave equation near the event horizon. Separating the variables from the standard wave equation, ingoing wave and outgoing wave can be obtained. Extending the wave equation from outside the event horizon into the inside by rotating $-\pi$ through the lower half of the complex plane, the thermal radiation spectra can be derived. Generalizing this method, many more works have been done [29-31].

References [32, 33] have also investigated the Hawking radiation by calculating the vacuum expectation values of the renormalized energy momentum tensor of spherically symmetric nonstationary black hole. These results are consistent with $[27,28]$. However all these research works were confined to the quantum thermal radiation of black hole only. In addition to the quantum thermal radiation, the importance of quantum nonthermal radiation of black hole has been studied by different authors in different types of space-time in the literatures [34-38].

The main aim of this paper is to investigate thermal radiation from Klein-Gordon equation, Maxwell's electromagnetic field equations, Dirac particles, and also nonthermal radiation of Hamilton-Jacobi equation in general nonstationary black hole. It gives the relationship between two kinds of radiation in the case of scalar particles. A new extra coupling effect is derived from the thermal radiation spectrum of Maxwell's field equations and Dirac particles. In Section 2, we derive the location of horizon in general nonstationary black hole using null surface condition and generalized tortoise coordinate transformation. In Section 3, adjusting the parameter $\kappa$, the Klein-Gordon equation is transformed into a wave equation near the event horizon in general nonstationary black hole. In Section 4, we derive asymptotic behaviors of first-order form of four equations and secondorder form of three equations from the Maxwell's electromagnetic field equations near the event horizon using generalized tortoise coordinate transformation (GTCT). In Section 5, the asymptotic behaviors of the first-order form of four equations and second-order form of two equations are deduced from the Dirac particles using the GTCT near the event horizon. In Section 6, the second-order form of Klein-Gordon equation, the three second-order forms of Maxwell's equations, and the two second-order forms of Dirac equations are transformed into single standard form of equation near the black hole event horizon. By separating the wave equation the chemical potential and the thermal radiation spectra can be obtained. In Section 7, the highest energy of nonthermal radiation is obtained from the relativistic Hamilton-Jacobi equation. In Section 8, we derive the expression of a new extra coupling effect which is absent from the thermal radiation spectrum of scalars particles. The relationship between thermal and nonthermal radiation in general nonstationary black hole is also established. Some conclusions are given in the last section.

\section{General Nonstationary Black Hole}

The line element describing the most general nonstationary black hole is given by

$$
d s^{2}=g_{\mu \nu} d x^{\mu} d x^{\nu}
$$

where we assume the retarded Eddington-Finkelstein coordinates $\left(x^{0}=u, x^{1}=r, x^{2}=\theta\right.$, and $\left.x^{3}=\phi\right)$ and make the conventions that all indices of Greek letters $\mu, \nu=0,1,2,3$ and all indices of Latin letters $j, k=0,2,3$. The event horizon in general nonstationary black hole can be characterized by null hypersurface condition: $F=F(u, r, \theta, \phi)=0$. The null hypersurface condition gives the position of horizon of stationary or nonstationary black hole [39]

$$
g^{\mu \nu} \partial_{\mu} F \partial_{\nu} F=0 .
$$

The location and the temperature of the horizon in a nonstationary black hole may be obtained by applying tortoise coordinate transformation. By using tortoise coordinate transformation of the form $r_{*}=r+(2 \kappa)^{-1} \ln \left(r-r_{h}\right)$, the KleinGordon equation, the Maxwell's electromagnetic field equations, and the Dirac particles can be combined into a standard form of wave equation in a nonstationary or stationary spacetime (where $\kappa$ is the surface gravity). The location of horizon may be assumed as functions of retarded time coordinate $u=t-r_{*}$ and different angles $\theta, \phi$. The space-time geometry outside the event horizon is described by tortoise coordinate only and, in this condition, $r_{*}$ approaches to positive infinity when tending to infinite point and $r_{*}$ tends to negative infinity at the event horizon. It is also assumed that the geometry of space-time in the general nonstationary black hole is symmetric about $\phi$-axis. According to [40-46], the generalized tortoise coordinate transformation is defined as

$$
\begin{aligned}
r_{*} & =r+\frac{1}{2 \kappa\left(u_{0}, \theta_{0}, \phi_{0}\right)} \ln \left\{\frac{r-r_{h}(u, \theta, \phi)}{r_{h}(u, \theta, \phi)}\right\}, \\
u_{*} & =u-u_{0}, \\
\theta_{*} & =\theta-\theta_{0}, \\
\phi_{*} & =\phi-\phi_{0},
\end{aligned}
$$

where $u_{0}, \theta_{0}$, and $\phi_{0}$ are the parameters under the tortoise coordinate transformation. From (3), we get

$$
\begin{aligned}
& \frac{\partial}{\partial r}=\frac{\partial}{\partial r_{*}}+\frac{1}{2 \kappa\left(r-r_{h}\right)} \frac{\partial}{\partial r_{*}}, \\
& \frac{\partial}{\partial x^{j}}=\frac{\partial}{\partial x_{*}^{j}}-\frac{r r_{h, j}}{2 \kappa r_{h}\left(r-r_{h}\right)} \frac{\partial}{\partial r_{*}},
\end{aligned}
$$




$$
\begin{aligned}
& \frac{\partial^{2}}{\partial r^{2}}=\frac{\left[2 \kappa\left(r-r_{h}\right)+1\right]^{2}}{\left[2 \kappa\left(r-r_{h}\right)\right]} \frac{\partial^{2}}{\partial r_{*}^{2}}-\frac{1}{2 \kappa\left(r-r_{h}\right)^{2}} \frac{\partial}{\partial r_{*}}, \\
& \frac{\partial^{2}}{\partial r \partial x^{j}}
\end{aligned}
$$$$
=\frac{\left[1+2 \kappa\left(r-r_{h}\right)\right]}{2 \kappa\left(r-r_{h}\right)} \frac{\partial^{2}}{\partial r_{*} \partial x_{*}^{j}}+\frac{r_{h, j}}{2 \kappa\left(r-r_{h}\right)^{2}} \frac{\partial}{\partial r_{*}}
$$$$
-r_{h} r r_{h, j} \frac{\left[1+2 \kappa\left(r-r_{h}\right)\right]}{\left[2 r_{h} \kappa\left(r-r_{h}\right)\right]^{2}} \frac{\partial^{2}}{\partial r_{*}^{2}},
$$$$
\frac{\partial^{2}}{\partial x^{j} \partial x^{k}}
$$

$$
\begin{aligned}
= & \frac{\partial^{2}}{\partial x_{*}^{j} \partial x_{*}^{k}}-\frac{r r_{h, j}}{2 \kappa\left(r-r_{h}\right)} \frac{\partial^{2}}{\partial r_{*} \partial x^{k}} \\
& -\frac{r r_{h, k}}{2 \kappa\left(r-r_{h}\right)} \frac{\partial^{2}}{\partial r_{*} \partial x^{j}}+\frac{r_{h}^{2} r_{h, j} r_{h, k}}{\left[2 \kappa r_{h}\left(r-r_{h}\right)\right]^{2}} \frac{\partial^{2}}{\partial r_{*}^{2}} \\
& -\frac{r}{2 \kappa} \frac{\left[\left(r-r_{h}\right) r_{h, j k}+r_{h, j} r_{h, k}\left(2 r_{h}-r\right)\right]}{\left[r_{h}\left(r-r_{h}\right)\right]^{2}} \frac{\partial}{\partial r_{*}} .
\end{aligned}
$$

Using (4) in (2), the horizon equation in general nonstationary black hole is obtained as

$$
g^{11}-2 g^{1 j} r_{h, j}+g^{j k} r_{h, j} r_{h, k}=0,
$$

where $r_{h, j}=\left(\partial r_{h} / \partial u, \partial r_{h} / \partial \theta, \partial r_{h} / \partial \phi\right) . r_{h, u}=\partial r_{h} / \partial u$ represents the evaporation rate in general nonstationary black hole near the event horizon. The event horizon is expanded gradually if $\partial r_{h} / \partial u>0$ (absorbing black hole), where, as $\partial r_{h} / \partial u<0$, the event horizon is contracted. In addition, $r_{h, \theta}=\partial r_{h} / \partial \theta$ and $r_{h, \phi}=\partial r_{h} / \partial \phi$ denote the rate of event horizon varying with angles and also describe the rotation effect of nonstationary black hole. $r_{h}$ is the location of event horizon and depends on retarded time $u_{0}$ and angular coordinates $\theta_{0}, \phi_{0}$, and also $\kappa \equiv \kappa\left(u_{0}, \theta_{0}, \phi_{0}\right)$ is an adjustable parameter that depends on retarded time and angular coordinates.

\section{Klein-Gordon Equation}

In this section, the asymptotic behavior of minimally electromagnetic coupling Klein-Gordon equation near the black hole will be discussed. The Klein-Gordon equation describes the explicit form of wave equation of the scalar particles with mass $\mu$ in curve space-time which is given by

$$
\frac{1}{\sqrt{-g}}\left[\frac{\partial}{\partial x^{a}} \sqrt{-g} g^{a b} \frac{\partial}{\partial x^{b}}\right] \Phi-\mu^{2} \Phi=0 .
$$

Using generalized tortoise coordinate transformation to (6) and subsequently multiplying by the factor $2 \kappa r_{h}(r-$ $\left.r_{h}\right) /\left[r_{h} g^{01}\left\{1+2 \kappa\left(r-r_{h}\right)\right\}-r g^{0 j} r_{h, j}\right]$ to both sides of (6) and finally taking limit near the event horizon as $r \rightarrow r_{h}\left(u_{0}, \theta_{0}\right.$, $\left.\phi_{0}\right), u \rightarrow u_{0}, \theta \rightarrow \theta_{0}$, and $\phi \rightarrow \phi_{0}$, the second-order form of wave equation is obtained as follows:

$$
\begin{aligned}
A \frac{\partial^{2} \Phi}{\partial r_{*}^{2}} & +2 \frac{\partial^{2} \Phi}{\partial r_{*} \partial u_{*}}+A_{1} \frac{\partial^{2} \Phi}{\partial r_{*} \partial \theta_{*}}+A_{2} \frac{\partial^{2} \Phi}{\partial r_{*} \partial \phi_{*}} \\
& +A_{3} \frac{\partial \Phi}{\partial r_{*}}=0,
\end{aligned}
$$

where

$$
\begin{aligned}
A & =\frac{g^{11} r_{h}^{2}\left[1+2 \kappa\left(r-r_{h}\right)\right]^{2}-2 g^{1 j} r r_{h} r_{h, \phi}\left[1+2 \kappa\left(r-r_{h}\right)\right]}{2 \kappa r_{h}\left(r-r_{h}\right)\left[r_{h} g^{01}\left\{1+2 \kappa\left(r-r_{h}\right\}-r g^{0 j} r_{h, j}\right]\right.}, \\
A_{1} & =2 \frac{g^{12}-g^{2 j} r_{h, j}}{\left(g^{01}-g^{0 j} r_{h, j}\right)}, \\
A_{2} & =2 \frac{g^{13}-g^{3 j} r_{h, j}}{\left(g^{01}-g^{0 j} r_{h, j}\right)} \\
A_{3} & =-\frac{\partial g^{11} / \partial r-2\left(\partial g^{1 j} / \partial r\right) r_{h, j}+g^{j k} r_{h, j k}}{\left(g^{01}-g^{0 j} r_{h, j}\right)} \\
& +\frac{g^{j k} r_{h, j} r_{h, k}}{r_{h}^{2}\left(g^{01}-g^{0 j} r_{h, j}\right)}\left(r_{h}-1\right) \\
& +\frac{1}{\sqrt{-g}\left(g^{01}-g^{0 j} r_{h, j}\right)}\left[\left(\sqrt{-g}, g^{1 v}+\sqrt{-g} g_{, v}^{1 v}\right)\right. \\
& -\left(\sqrt{-g} g^{0 v}+\sqrt{-g} g_{, v}^{0 \nu}\right)-\left(\sqrt{-g} g_{, \nu}^{2 v}+\sqrt{-g} g_{, v}^{2 v}\right) \\
& \left.-\left(\sqrt{-g} g^{3 v}+\sqrt{-g} g_{, v}^{3 v}\right)\right] .
\end{aligned}
$$

By adjusting parameter $\kappa$, the coefficient of $\partial^{2} \Phi / \partial r_{*}^{2}$ is assumed to be unity near the event horizon, and we get

$$
\begin{aligned}
\lim _{r \rightarrow r_{h}} & \frac{1}{2 \kappa r_{h}\left(r-r_{h}\right)}\left[g^{11} r_{h}^{2}\left\{1+2 \kappa\left(r-r_{h}\right)\right\}^{2}\right. \\
& \left.-2 g^{1 j} r r_{h} r_{h, \phi}\left\{1+2 \kappa\left(r-r_{h}\right)\right\}+r^{2} g^{j k} r_{h, j} r_{h, k}\right] \\
& =r_{h}\left(g^{01}-g^{0 j} r_{h, j}\right) .
\end{aligned}
$$

It is also observed that, in the left hand side of (9), both numerator and denominator tend to zero near the event horizon $r=r_{h}$. Hence (9) is an indeterminate form of $0 / 0$. Using L'Hospital rule and using (5), the surface gravity is obtained from the Klein-Gordon scalar particles as follows:

$$
\begin{aligned}
\kappa= & \frac{\partial g^{11} / \partial r-2\left(\partial g^{1 j} / \partial r\right) r_{h, j}+\left(\partial g^{j k} / \partial r\right) r_{h, j} r_{h, k}}{2\left[g^{01}-2 g^{11}+\left(2 g^{1 j}-g^{0 j}\right) r_{h, j}\right]} \\
& +\frac{g^{j k} r_{h, j} r_{h, k}-g^{1 j} r_{h, j}}{r_{h}\left[g^{01}-2 g^{11}+\left(2 g^{1 j}-g^{0 j}\right) r_{h, j}\right]} .
\end{aligned}
$$

\section{Maxwell's Electromagnetic Field Equations}

To write the explicit form of Maxwell's electromagnetic field equations in Newman-Penrose formalism [47], the following 
complex null tetrad vectors $\ell, n, m$, and $\bar{m}$ are chosen at each point in four-dimensional space, where $\ell$ and $n$ are a pair of real null tetrad vectors and $m$ and $\bar{m}$ are a pair of complex null tetrad vectors. They are required to satisfy the following conditions:

$$
\begin{aligned}
\ell_{\nu} \ell^{\nu} & =n_{\nu} n^{\nu}=m_{\nu} m^{\nu}=\bar{m}_{\nu} \bar{m}^{v}=0, \\
\ell_{\nu} n^{\nu} & =-m_{\nu} \bar{m}^{\nu}=1, \\
\ell_{\nu} m^{\nu} & =\ell_{\nu} \bar{m}^{\nu}=n_{\nu} m^{\nu}=n_{\nu} \bar{m}^{v}=0, \\
g^{\mu \nu} & =\ell^{\mu} n^{\nu}+\ell^{\nu} n^{\mu}-m^{\mu} \bar{m}^{\nu}-m^{\nu} \bar{m}^{\mu}, \\
g_{\mu \nu} & =\ell_{\mu} n_{\nu}+\ell_{\nu} n_{\mu}-m_{\mu} \bar{m}_{\nu}-m_{\nu} \bar{m}_{\mu}
\end{aligned}
$$

and corresponding directional derivatives are given by

$$
\begin{aligned}
& D=\ell^{\nu} \frac{\partial}{\partial x^{\mu}}, \\
& \Delta=n^{\nu} \frac{\partial}{\partial x^{\mu}} \\
& \delta=m^{\nu} \frac{\partial}{\partial x^{\mu}}, \\
& \bar{\delta}=\bar{m}^{\nu} \frac{\partial}{\partial x^{\mu}} .
\end{aligned}
$$

The dynamical behavior of spin-1 particles in curve spacetime is given by four coupled Maxwell's electromagnetic field equations expressed in Newman-Penrose formalism [48] as follows:

$$
\begin{gathered}
D \phi_{1}-\bar{\delta} \phi_{0}-(\pi-2 \alpha) \phi_{0}-2 \rho \phi_{1}+\kappa \phi_{2}=0, \\
\delta \phi_{2}-\nabla \phi_{1}+\nu \phi_{0}-2 \mu \phi_{1}-(\tau-2 \beta) \phi_{2}=0, \\
\delta \phi_{1}-\nabla \phi_{0}-(\mu-2 \gamma) \phi_{0}-2 \tau \phi_{1}+\sigma \phi_{2}=0, \\
D \phi_{2}-\bar{\delta} \phi_{1}+\lambda \phi_{0}-2 \pi \phi_{1}-(\rho-2 \varepsilon) \phi_{2}=0
\end{gathered}
$$

where $\phi_{0}, \phi_{1}$, and $\phi_{2}$ are the four components of Maxwell's spinor in the Newman-Penrose formalism. $\epsilon, \rho, \pi, \alpha, \beta, \mu, \tau$, and $\gamma$ are spin coefficients introduced by Newman and Penrose, and they are given by

$$
\begin{aligned}
& \rho=\ell_{\mu ; \nu} m^{\mu} \bar{m}^{\nu}, \\
& \pi=-n_{\mu ; \nu} \bar{m}^{\mu} \ell^{\nu}, \\
& \tau=\ell_{\mu ; \nu} m^{\mu} n^{\nu}, \\
& \alpha=\frac{1}{2}\left(\ell_{\mu ; \nu} n^{\mu} \bar{m}^{v}-m_{\mu ; \nu} \bar{m}^{\mu} \bar{m}^{v}\right), \\
& \beta=\frac{1}{2}\left(\ell_{\mu ; \nu} n^{\mu} m^{\nu}-m_{\mu ; \nu} \bar{m}^{\mu} m^{\nu}\right), \\
& \gamma=\frac{1}{2}\left(\ell_{\mu ; \nu} n^{\mu} n^{\nu}-m_{\mu ; \nu} \bar{m}^{\mu} n^{\nu}\right), \\
& \epsilon=\frac{1}{2}\left(\ell_{\mu ; \nu} n^{\mu} \ell^{\nu}-m_{\mu ; \nu} \bar{m}^{\mu} \ell^{\nu}\right),
\end{aligned}
$$

where $\bar{\alpha}, \bar{\beta}, \bar{\gamma}, \bar{\tau}, \bar{\epsilon}, \bar{\pi}, \bar{\mu}$, and $\bar{\rho}$ are complex conjugates of $\alpha, \beta$, $\gamma, \tau, \epsilon, \pi, \mu$, and $\rho$. From (13), the three second-order forms of Maxwell's equations for $\left(\phi_{0}, \phi_{1}, \phi_{2}\right)$ components are given by

$$
\begin{aligned}
& D \nabla \phi_{0}-\delta \bar{\delta} \phi_{0}+(\mu-2 \gamma) D \phi_{0}-(\pi-2 \alpha) \delta \phi_{0} \\
& -2 \rho \nabla \phi_{0}+2 \tau \bar{\delta} \phi_{0}+\left(m^{\nu} \ell_{, v}^{\mu}-\bar{m}_{\nu}^{\mu} \ell^{\nu}\right) \frac{\partial \phi_{1}}{\partial x^{\mu}}+\kappa \nabla \phi_{2} \\
& -\sigma \bar{\delta} \phi_{2}=0 . \\
& \nabla D \phi_{1}-\bar{\delta} \delta \phi_{1}-2 \rho \nabla \phi_{1}+(\mu-2 \gamma) D \phi_{1}+2 \tau \bar{\delta} \phi_{1} \\
& -(\pi-2 \alpha) \delta \phi_{1}+\left(\bar{m}^{\nu} n_{, v}^{\mu}-n^{\nu} \bar{m}_{, \nu}^{\mu}\right) \frac{\partial \phi_{0}}{\partial x^{\mu}}+\kappa \nabla \phi_{2} \\
& -\sigma \bar{\delta} \phi_{2}=0 . \\
& \bar{\delta} \delta \phi_{2}-\nabla D \phi_{2}-(\tau-2 \beta) \bar{\delta} \phi_{2}+2 \pi \delta \phi_{2}+(\rho-2 \epsilon) \nabla \phi_{2} \\
& -2 \mu D \phi_{2}+\left(\bar{m}_{\nu}^{\mu} n^{\nu}-n_{, v}^{\mu} \bar{m}^{\nu}\right) \frac{\partial \phi_{1}}{\partial x^{\mu}}-\lambda \nabla \phi_{0} \\
& +\nu \bar{\delta} \phi_{0}=0 .
\end{aligned}
$$

References $[49,50]$ have shown that (13) cannot be decoupled except only for the stationary black hole space-time. For studying the thermal radiation in general nonstationary black hole, the asymptotic behavior of the first-order and secondorder form of (13) near the event horizon $r=r_{h}$ will be considered. Then, after taking the limit as $r \rightarrow r_{h}\left(u_{0}, \theta_{0}, \phi_{0}\right)$, $u \rightarrow u_{0}, \theta \rightarrow \theta_{0}$, and $\phi \rightarrow \phi_{0}$, the first-order forms of Maxwell's equations near the event horizon are as follows:

$$
\begin{aligned}
& \left(\ell^{1}-\ell^{j} r_{h, j}\right) \frac{\partial \phi_{1}}{\partial r_{*}}-\left(\bar{m}^{1}-\bar{m}^{j} r_{h, j}\right) \frac{\partial \phi_{0}}{\partial r_{*}}=0, \\
& \left(m^{1}-m^{j} r_{h, j}\right) \frac{\partial \phi_{2}}{\partial r_{*}}-\left(n^{1}-n^{j} r_{h, j}\right) \frac{\partial \phi_{1}}{\partial r_{*}}=0, \\
& \left(m^{1}-m^{k} r_{h, k}\right) \frac{\partial \phi_{1}}{\partial r_{*}}-\left(n^{1}-n^{j} r_{h, k}\right) \frac{\partial \phi_{0}}{\partial r_{*}}=0, \\
& \left(\ell^{1}-\ell^{k} r_{h, k}\right) \frac{\partial \phi_{2}}{\partial r_{*}}-\left(\bar{m}^{1}-\bar{m}^{k} r_{h, k}\right) \frac{\partial \phi_{1}}{\partial r_{*}}=0 .
\end{aligned}
$$

We assume that the three derivatives $\partial \phi_{0} / \partial r_{*}, \partial \phi_{1} / \partial r_{*}$, and $\partial \phi_{2} / \partial r_{*}$ in (18) are nonzero. Then nontrivial solutions for $\phi_{0}, \phi_{1}$, and $\phi_{2}$ can be obtained if the determinant of their coefficients is zero, which will give the horizon equation like the null surface condition (5). The importance of (18) is to eliminate the crossing terms involved in the second-order form of Maxwell's equations near the event horizon.

Utilizing the generalized coordinate transformation (3) to (15), (16), and (17) and subsequently multiplying by the factor $2 \kappa r_{h}\left(r-r_{h}\right) /\left[r_{h} g^{01}\left\{1+2 \kappa\left(r-r_{h}\right)\right\}-r g^{0 j} r_{h, j}\right]$ to both sides of three second-order equations for the coefficients $\partial^{2} \phi_{0} / \partial r_{*} \partial u_{*}, \partial^{2} \phi_{1} / \partial r_{*} \partial u_{*}$, and $\partial^{2} \phi_{2} / \partial r_{*} \partial u_{*}$ to be 2 and finally taking the limit of $r \rightarrow r_{h}\left(u_{0}, \theta_{0}, \phi_{0}\right), u \rightarrow u_{0}, \theta \rightarrow \theta_{0}$, and $\phi \rightarrow \phi_{0}$, then the three second-order forms of Maxwell's 
electromagnetic field equations near the event horizon can be expressed as follows:

$$
\begin{aligned}
I \frac{\partial^{2} \phi_{0}}{\partial r_{*}^{2}} & +2 \frac{\partial^{2} \phi_{0}}{\partial r_{*} \partial u_{*}}+B_{1} \frac{\partial^{2} \phi_{0}}{\partial r_{*} \partial \theta_{*}}+B_{2} \frac{\partial^{2} \phi_{0}}{\partial r_{*} \partial \phi_{*}} \\
& +\left(B_{3}+2 i B_{4}\right) \frac{\partial \phi_{0}}{\partial r_{*}}=0,
\end{aligned}
$$

where

$$
\begin{aligned}
& I=\frac{g^{11} r_{h}^{2}\left\{1+2 \kappa\left(r-r_{h}\right)\right\}^{2}-2 g^{1 j} r r_{h} r_{h, \phi}\left\{1+2 \kappa\left(r-r_{h}\right)\right\}}{2 \kappa r_{h}\left(r-r_{h}\right)\left[r_{h} g^{01}\left\{1+2 \kappa\left(r-r_{h}\right)\right\}-r g^{0 j} r_{h, j}\right]}, \\
& B_{1}=2 \frac{\left(g^{12}-g^{2 j} r_{h, j}\right)}{\left(g^{01}-g^{0 j} r_{h, j}\right)}, \\
& B_{2}=2 \frac{\left(g^{13}-g^{3 j} r_{h, j}\right)}{\left(g^{01}-g^{0 j} r_{h, j}\right)} \\
& B_{3}=-\frac{\partial g^{11} / \partial r-2\left(\partial g^{1 j} / \partial r\right) r_{h, j}+g^{j k} r_{h, j k}}{\left(g^{01}-g^{0 j} r_{h, j}\right)} \\
& +\frac{g^{j k} r_{h, j} r_{h, k}}{r_{h}^{2}\left(g^{01}-g^{0 j} r_{h, j}\right)}\left(r_{h}-1\right), \\
& B_{4}=\frac{-i}{\left(g^{01}-g^{0 j} r_{h, j}\right)}\left[\ell^{v}\left(n_{, v}^{1}-n_{, v}^{j} r_{h, j}\right)-m^{\nu}\left(\ell_{, v}^{1}-\ell_{, v}^{j} r_{h, j}\right)\right. \\
& -2 \rho\left(n^{1}-n^{j} r_{h, j}\right)+(\mu-2 \gamma)\left(\ell_{, v}^{1}-\ell_{, v}^{j} r_{h, j}\right)-(\pi-2 \alpha) \\
& \cdot\left(m^{1}-m^{j} r_{h, j}\right)+2 \tau\left(\bar{m}^{1}-\bar{m}^{j} r_{h, j}\right) \\
& +\frac{\left(\bar{m}^{1}-\bar{m}^{j} r_{h, j}\right)}{\left(\ell^{1}-\ell^{j} r_{h, j}\right)}\left\{m^{v}\left(\ell_{, v}^{1}-\ell_{, v}^{j} r_{h, j}\right)-\ell^{v}\left(\bar{m}_{, v}^{1}-\bar{m}^{j} r_{h, j}\right)\right\} \\
& -\left\{\sigma\left(\bar{m}^{1}-\bar{m}^{j} r_{h, j}\right)-\kappa\left(n^{1}-n^{j} r_{h, j}\right)\right\} \\
& \left.\cdot \frac{\left(n^{1}-n^{j} r_{h, j}\right)\left(\bar{m}^{1}-\bar{m}^{j} r_{h, j}\right)}{\left(m^{1}-m^{j} r_{h, j}\right)\left(\ell^{1}-\ell^{j} r_{h, j}\right)}\right] \text {, } \\
& I \frac{\partial^{2} \phi_{1}}{\partial r_{*}^{2}}+2 \frac{\partial^{2} \phi_{1}}{\partial r_{*} \partial u_{*}}+B_{1} \frac{\partial^{2} \phi_{1}}{\partial r_{*} \partial \theta_{*}}+B_{2} \frac{\partial^{2} \phi_{1}}{\partial r_{*} \partial \phi_{*}}+\left(B_{3}\right. \\
& \left.+2 i \widetilde{B}_{4}\right) \frac{\partial \phi_{1}}{\partial r_{*}}=0,
\end{aligned}
$$

where

$$
\begin{aligned}
\widetilde{B}_{4} & =\frac{-i}{\left(g^{01}-g^{0 j} r_{h, j}\right)}\left[-2 \rho\left(n^{1}-n^{j} r_{h, j}\right)+(\mu-2 \gamma)\right. \\
& \left(\ell^{1}-\ell^{j} r_{h, j}\right)+2 \tau\left(\bar{m}^{1}-\bar{m}^{j} r_{h, j}\right)-(\pi-2 \alpha)\left(m^{1}\right. \\
& \left.-m^{j} r_{h, j}\right)+n^{v}\left(\ell_{, v}^{1}-\ell_{, v}^{j} r_{h, j}\right)-\bar{m}^{v}\left(m_{, v}^{1}-m_{, v}^{j} r_{h, j}\right) \\
& +\frac{\left(\ell^{1}-\ell^{j} r_{h, j}\right)}{\left(\bar{m}^{1}-\bar{m}^{j} r_{h, j}\right)}\left\{\bar{m}^{v}\left(n_{, v}^{1}-n_{, v}^{j} r_{h, j}\right)\right.
\end{aligned}
$$

$$
\begin{aligned}
& \left.-n^{v}\left(\bar{m}_{, v}^{1}-\bar{m}_{, v}^{j} r_{h, j}\right)\right\}+\left\{-\sigma\left(\bar{m}^{1}-\bar{m}^{j} r_{h, j}\right)\right. \\
& \left.\left.+\kappa\left(n^{1}-n^{j} r_{h, j}\right)\right\} \frac{\left(n^{1}-n^{j} r_{h, j}\right)}{\left(m^{1}-m^{j} r_{h, j}\right)}\right],
\end{aligned}
$$

$$
\begin{aligned}
& I \frac{\partial^{2} \phi_{2}}{\partial r_{*}^{2}}+2 \frac{\partial^{2} \phi_{2}}{\partial r_{*} \partial u_{*}}+B_{1} \frac{\partial^{2} \phi_{2}}{\partial r_{*} \partial \theta_{*}}+B_{2} \frac{\partial^{2} \phi_{2}}{\partial r_{*} \partial \phi_{*}}+\left(B_{3}\right. \\
& \left.\quad+2 i \bar{B}_{4}\right) \frac{\partial \phi_{2}}{\partial r_{*}}=0,
\end{aligned}
$$

where

$$
\begin{aligned}
\bar{B}_{4} & =\frac{-i}{\left(g^{01}-g^{0 j} r_{h, j}\right)}\left[n^{\nu}\left(\ell_{, v}^{1}-\ell_{, v}^{j} r_{h, j}\right)-\bar{m}^{v}\left(m_{, v}^{1}\right.\right. \\
& \left.-m_{, v}^{j} r_{h, j}\right)-2 \pi\left(m_{, v}^{1}-m_{, v}^{j} r_{h, j}\right)+(\tau-2 \beta)\left(\bar{m}_{, v}^{1}\right. \\
& \left.-\bar{m}_{, v}^{j}\right)-2(\rho-2 \epsilon)\left(n_{, v}^{1}-n_{, v}^{j} r_{h, j}\right)+2 \mu\left(\ell_{, v}^{1}\right. \\
- & \left.\ell_{, v}^{j} r_{h, j}\right)+\frac{\left(\ell^{1}-\ell^{j} r_{h, j}\right)}{\left(\bar{m}^{1}-\bar{m}^{j} r_{h, j}\right)} \\
& \cdot \frac{\left(m^{1}-m^{j} r_{h, j}\right)}{\left(n^{1}-n^{j} r_{h, j}\right)}\left\{\lambda\left(n_{, v}^{1}-n_{, v}^{j} r_{h, j}\right)\right. \\
& \left.-v\left(\bar{m}_{, v}^{1}-\bar{m}_{, v}^{j} r_{h, j}\right)\right\}-2\left\{n^{\nu}\left(\bar{m}^{1}-\bar{m}^{j} r_{h, j}\right)\right. \\
& \left.\left.-\bar{m}^{v}\left(n_{, v}^{1}-n_{, v}^{j} r_{h, j}\right)\right\} \frac{\left(m^{1}-m^{j} r_{h, j}\right)}{\left(n^{1}-n^{j} r_{h, j}\right)}\right] .
\end{aligned}
$$

We assume the value of $I$ approaches unity near the event horizon, and then we get

$$
\begin{aligned}
& \lim _{r \rightarrow r_{h}} \frac{g^{11} r_{h}^{2}\left\{1+2 \kappa\left(r-r_{h}\right)\right\}^{2}-2 g^{1 j} r r_{h} r_{h, \phi}\left\{1+2 \kappa\left(r-r_{h}\right)\right\}}{2 \kappa r_{h}\left(r-r_{h}\right)\left[r_{h} g^{01}\left\{1+2 \kappa\left(r-r_{h}\right)\right\}-r g^{0 j} r_{h, j}\right]} \\
& \quad=1,
\end{aligned}
$$

which is an indeterminate form of $0 / 0$ and, applying L'Hospital rule, the surface gravity due to the Dirac particles is given by

$$
\begin{aligned}
\kappa= & \frac{\partial g^{11} / \partial r-2\left(\partial g^{1 j} / \partial r\right) r_{h, j}+\left(\partial g^{j k} / \partial r\right) r_{h, j} r_{h, k}}{2\left[g^{01}-2 g^{11}+\left(2 g^{1 j}-g^{0 j}\right) r_{h, j}\right]} \\
& +\frac{g^{j k} r_{h, j} r_{h, k}-g^{1 j} r_{h, j}}{r_{h}\left[g^{01}-2 g^{11}+\left(2 g^{1 j}-g^{0 j}\right) r_{h, j}\right]},
\end{aligned}
$$

which is equal to the surface gravity derived from KleinGordon scalar particle given by (10). 


\section{Dirac Equations}

The four couples of Dirac equations [51] expressed in Newman-Penrose formalism are given by

$$
\begin{aligned}
& (D+\epsilon-\rho) f_{1}+(\bar{\delta}+\pi-\alpha) f_{2}-\frac{i \mu_{0} g_{1}}{\sqrt{2}}=0, \\
& (\nabla+\mu-\gamma) f_{2}+(\delta+\beta-\tau) F_{1}-\frac{i \mu_{0} g_{2}}{\sqrt{2}}=0, \\
& (\nabla+\bar{\mu}-\bar{\gamma}) g_{1}-(\bar{\delta}+\bar{\beta}-\bar{\tau}) g_{2}-\frac{i \mu_{0} f_{1}}{\sqrt{2}}=0, \\
& (D+\bar{\epsilon}-\bar{\rho}) g_{2}-(\delta+\bar{\pi}-\bar{\alpha}) g_{1}-\frac{i \mu_{0} f_{2}}{\sqrt{2}}=0,
\end{aligned}
$$

where $D, \nabla, \delta$, and $\bar{\delta}$ are the directional derivatives given by (12) and $\epsilon, \rho, \pi, \alpha, \beta, \mu, \tau$, and $\gamma$ are spin coefficients and also $\mu_{0}$ is the mass of the Dirac particles. $f_{1}, f_{2}, g_{1}$, and $g_{2}$ are the four components of Dirac spinor in the NewmanPenrose formalism. Equations (27) can be decoupled only for the stationary black hole space-time. From (27) the secondorder forms of Dirac equations for the components $\left(f_{1}, f_{2}\right)$ are given by

$$
\begin{aligned}
& -2(\nabla+\bar{\mu}-\bar{\gamma})\left[(D+\epsilon-\rho) f_{1}+(\bar{\delta}+\phi-\alpha) f_{2}\right] \\
& +2(\bar{\delta}+\bar{\beta}-\bar{\tau})\left[(\nabla+\mu-\gamma) f_{2}+(\delta+\beta-\tau) f_{1}\right] \\
& -\mu_{0}^{2} f_{1}=0, \\
& -2(D+\bar{\epsilon}-\bar{\rho})\left[(\nabla+\mu-\gamma) f_{2}+(\delta+\beta-\tau) f_{1}\right] \\
& +2(\delta+\bar{\pi}-\bar{\alpha})\left[(D+\epsilon-\rho) f_{1}+(\bar{\delta}+\pi-\alpha) f_{2}\right] \\
& -\mu_{0}^{2} f_{2}=0 .
\end{aligned}
$$

Applying generalized tortoise coordinate transformation to (27), after taking limit $r \rightarrow r_{h}\left(u_{0}, \theta_{0}, \phi_{0}\right), u \rightarrow u_{0}, \theta \rightarrow \theta_{0}$, and $\phi \rightarrow \phi_{0}$, the first-order forms of Dirac equations near the event horizon are given by

$$
\begin{aligned}
& \frac{\partial f_{1}}{\partial r_{*}}=\frac{\bar{m}^{1}-\bar{m}^{j} r_{h, j}}{\ell^{j} r_{h, j}-\ell^{1}} \frac{\partial f_{2}}{\partial r_{*}}, \\
& \frac{\partial f_{2}}{\partial r_{*}}=\frac{m^{1}-m^{j} r_{h, j}}{n^{j} r_{h, j}-n^{1}} \frac{\partial f_{1}}{\partial r_{*}} \\
& \frac{\partial g_{2}}{\partial r_{*}}=\frac{m^{1}-m^{k} r_{h, k}}{\ell^{1}-\ell^{k} r_{h, k}} \frac{\partial g_{1}}{\partial r_{*}} \\
& \frac{\partial g_{1}}{\partial r_{*}}=\frac{\bar{m}^{1}-\bar{m}^{k} r_{h, k}}{n^{1}-n^{k} r_{h, k}} \frac{\partial g_{2}}{\partial r_{*}}
\end{aligned}
$$

In order to study Hawking thermal radiation from Dirac particles, we should consider the asymptotic behavior of (27) near the black hole horizon. The nontrivial solutions for $f_{1}, f_{2}, g_{1}$, and $g_{2}$ can be obtained if the four derivatives $\partial f_{1} / \partial r_{*}, \partial f_{2} / \partial r_{*}, \partial g_{1} / \partial r_{*}$, and $\partial g_{2} / \partial r_{*}$ in (30) are nonzero. Equations (30) may be used to eliminate the crossing terms that appeared in the second-order form of Dirac equation near the horizon.

Applying generalized tortoise coordinate transformation to (28) and (29), via some arrangement, and multiplying by the factor $2 \kappa r_{h}\left(r-r_{h}\right) /\left[r_{h} g^{01}\left\{1+2 \kappa\left(r-r_{h}\right)\right\}-r g^{0 j} r_{h, j}\right]$ to both sides of the two second-order forms of Dirac equations for the coefficients $\partial^{2} f_{1} / \partial r_{*} \partial u_{*}$ and $\partial^{2} f_{2} / \partial r_{*} \partial u_{*}$ to be 2 and taking the limit of $r \rightarrow r_{h}\left(u_{0}, \theta_{0}, \phi_{0}\right), u \rightarrow u_{0}, \theta \rightarrow \theta_{0}$, and $\phi \rightarrow \phi_{0}$, the two second-order forms of Dirac equations can be written as follows:

$$
\begin{aligned}
& Q \frac{\partial^{2} f_{1}}{\partial r_{*}^{2}}+2 \frac{\partial^{2} f_{1}}{\partial r_{*} \partial u_{*}}+C_{1} \frac{\partial^{2} f_{1}}{\partial r_{*} \partial \theta_{*}}+C_{2} \frac{\partial^{2} f_{1}}{\partial r_{*} \partial \phi_{*}} \\
& \quad+\left(C_{3}+2 i C_{4}\right) \frac{\partial f_{1}}{\partial r_{*}}=0,
\end{aligned}
$$

where

$$
\begin{aligned}
& Q=\frac{g^{11} r_{h}^{2}\left\{1+2 \kappa\left(r-r_{h}\right)\right\}^{2}-2 g^{1 j} r r_{h} r_{h, \phi}\left\{1+2 \kappa\left(r-r_{h}\right)\right\}}{2 \kappa r_{h}\left(r-r_{h}\right)\left[r_{h} g^{01}\left\{1+2 \kappa\left(r-r_{h}\right)\right\}-r g^{0 j} r_{h, j}\right]} \\
& C_{1}=2 \frac{\left(g^{12}-g^{2 j} r_{h, j}\right)}{\left(g^{01}-g^{0 j} r_{h, \mathrm{j}}\right)} \\
& C_{2}=2 \frac{\left(g^{13}-g^{3 j} r_{h, j}\right)}{\left(g^{01}-g^{0 j} r_{h, j}\right)} \\
& C_{3}=-\frac{\partial g^{11} / \partial r-2\left(\partial g^{1 j} / \partial r\right) r_{h, j}+g^{j k} r_{h, j k}}{\left(g^{01}-g^{0 j} r_{h, j}\right)} \\
& +\frac{g^{j k} r_{h, j} r_{h, k}}{r_{h}^{2}\left(g^{01}-g^{0 j} r_{h, j}\right)}\left(r_{h}-1\right) \text {, } \\
& C_{4}=\frac{-i}{\left(g^{01}-g^{0 j} r_{h, j}\right)}\left[-(\beta-\tau+\bar{\beta}-\bar{\tau})\left(\bar{m}^{1}-\bar{m}^{j} r_{h, j}\right)\right. \\
& +(\epsilon-\rho)\left(n^{1}-n^{j} r_{h, j}\right)+(\bar{\mu}-\bar{\gamma})\left(\ell^{1}-\ell^{j} r_{h, j}\right) \\
& -\frac{\left(\ell^{1}-\ell^{j} r_{h, j}\right)}{\left(\bar{m}^{1}-\bar{m}^{j} r_{h, j}\right)}\left\{-\bar{m}^{v}\left(n_{, v}^{1}-n_{, v}^{j}\right)+n^{v}\left(\bar{m}^{1}-\bar{m}_{, v}^{j}\right)\right\} \\
& -(\bar{\mu}-\bar{\gamma}-\mu+\gamma)\left(\ell^{1}-\ell^{j} r_{h, j}\right) \\
& \left.-(\pi-\alpha-\bar{\beta}+\bar{\tau}) \frac{\left(\ell^{1}-\ell^{j} r_{h, j}\right)^{2}}{\left(\bar{m}^{1}-\bar{m}^{j} r_{h, j}\right)}\right], \\
& Q \frac{\partial^{2} f_{2}}{\partial r_{*}^{2}}+2 \frac{\partial^{2} f_{2}}{\partial r_{*} \partial u_{*}}+C_{1} \frac{\partial^{2} f_{2}}{\partial r_{*} \partial \theta_{*}}+C_{2} \frac{\partial^{2} f_{2}}{\partial r_{*} \partial \phi_{*}}+\left(C_{3}\right. \\
& \left.+2 i \bar{C}_{4}\right) \frac{\partial f_{2}}{\partial r_{*}}=0
\end{aligned}
$$


where

$$
\begin{aligned}
\bar{C}_{4} & =\frac{-i}{g^{01}-g^{0 j} r_{h, j}}\left[(\mu-\gamma)\left(\ell^{1}-\ell^{j} r_{h, j}\right)-(\pi-\alpha)\right. \\
& \cdot\left(m^{1}-m^{j} r_{h, j}\right)+\ell^{\nu}\left(n_{, v}^{1}-n_{, v}^{j}\right)+(\bar{\epsilon}-\bar{\rho})\left(n^{1}\right. \\
& \left.-n^{j} r_{h, j}\right)+\ell^{\nu}\left(n_{, v}^{1}-n_{, v}^{j}\right)-m^{\nu}\left(\bar{m}^{1}-\bar{m}_{, v}^{j}\right)-(\bar{\epsilon}-\bar{\rho} \\
& -\epsilon+\rho)\left(n_{, v}^{1}-n_{, v}^{j}\right)-\ell^{\nu} \frac{\left(m_{, \nu}^{1}-m_{, v}^{j}\right)}{\left(\bar{m}^{1}-\bar{m}_{h, j}^{j}\right)}\left(n^{1}-n^{j} r_{h, j}\right) \\
& +\left\{m^{\nu} \frac{\left(\ell_{, v}^{1}-\ell_{, v}^{j}\right)}{\left(m^{1}-m^{j} r_{h, j}\right)}\right. \\
& \left.\left.-(\beta-\tau-\bar{\pi}+\bar{\alpha}) \frac{\left(\ell^{1}-\ell^{j} r_{h, j}\right)}{\left(m^{1}-m^{j} r_{h, j}\right)}\right\}\left(n^{1}-n^{j} r_{h, j}\right)\right],
\end{aligned}
$$

and when $Q$ approaches unity, we obtain

$$
\begin{aligned}
& \lim _{r \rightarrow r_{h}} \frac{g^{11} r_{h}^{2}\left\{1+2 \kappa\left(r-r_{h}\right)\right\}^{2}-2 g^{1 j} r r_{h} r_{h, \phi}\left\{1+2 \kappa\left(r-r_{h}\right)\right\}}{2 \kappa r_{h}\left(r-r_{h}\right)\left[r_{h} g^{01}\left\{1+2 \kappa\left(r-r_{h}\right)\right\}-r g^{0 j} r_{h, j}\right]} \\
& \quad=1 .
\end{aligned}
$$

Equation (35) is a $0 / 0$ indeterminate form. By applying L'Hospital rule near the black hole event horizon, the surface gravity due to Dirac particle is given by

$$
\begin{aligned}
\kappa= & \frac{\partial g^{11} / \partial r-2\left(\partial g^{1 j} / \partial r\right) r_{h, j}+\left(\partial g^{j k} / \partial r\right) r_{h, j} r_{h, k}}{2\left[g^{01}-2 g^{11}+\left(2 g^{1 j}-g^{0 j}\right) r_{h, j}\right]} \\
& +\frac{g^{j k} r_{h, j} r_{h, k}-g^{1 j} r_{h, j}}{r_{h}\left[g^{01}-2 g^{11}+\left(2 g^{1 j}-g^{0 j}\right) r_{h, j}\right]},
\end{aligned}
$$

which is the same as (10) and (26), the surface gravities derived from Klein-Gordon scalar particles, and Maxwell's electromagnetic field equations.

\section{Thermal Radiation Spectrum}

To investigate the thermal radiation spectrum in general nonstationary black hole, we combine the second-order form of Klein-Gordon equation (7), the three second-order forms Maxwell's equations (19), (21), and (23), and the two secondorder forms of Dirac equations (31) and (33) as single form of wave equation near the event horizon $r=r_{h}$ as follows:

$$
\begin{aligned}
\frac{\partial^{2} \Psi}{\partial r_{*}^{2}} & +2 \frac{\partial^{2} \Psi}{\partial r_{*} \partial u_{*}}+L_{1} \frac{\partial^{2} \Psi}{\partial r_{*} \partial \theta_{*}}+L_{2} \frac{\partial^{2} \Psi}{\partial r_{*} \partial \phi_{*}} \\
& +\left(L_{3}+2 i L_{4}\right) \frac{\partial \Psi}{\partial r_{*}}=0,
\end{aligned}
$$

where

$$
\begin{aligned}
& L_{1}=2 \frac{\left(g^{12}-g^{2 j} r_{h, j}\right)}{\left(g^{01}-g^{0 j} r_{h, j}\right)}, \\
& L_{2}=2 \frac{\left(g^{13}-g^{3 j} r_{h, j}\right)}{\left(g^{01}-g^{0 j} r_{h, j}\right)} .
\end{aligned}
$$

Equation (37) may be assumed as standard form of wave equation in general nonstationary black hole near the horizon $r=r_{h}$. It includes Klein-Gordon equation, Maxwell's electromagnetic field equations, and Dirac equations with different coefficient of constant terms.

For example, when $(\Psi=\Phi)$ for the Klein-Gordon equation, (37) gives the following constants:

$$
\begin{aligned}
L_{3} & =-\frac{\partial g^{11} / \partial r-2\left(\partial g^{1 j} / \partial r\right) r_{h, j}+g^{j k} r_{h, j k}}{\left(g^{01}-g^{0 j} r_{h, j}\right)} \\
& +\frac{g^{j k} r_{h, j} r_{h, k}}{r_{h}^{2}\left(g^{01}-g^{0 j} r_{h, j}\right)}\left(r_{h}-1\right) \\
& +\frac{1}{\sqrt{-g}\left(g^{01}-g^{0 j} r_{h, j}\right)}\left[\left(\sqrt{-g}, g^{1 v}+\sqrt{-g} g_{, v}^{1 v}\right)\right. \\
& -\left(\sqrt{-g}, g^{0 v}+\sqrt{-g} g_{, \nu}^{0 v}\right)-\left(\sqrt{-g}, g^{2 v}+\sqrt{-g} g_{, \nu}^{2 v}\right) \\
& \left.-\left(\sqrt{-g} g_{,} g^{3 v}+\sqrt{-g} g_{, v}^{3 v}\right)\right],
\end{aligned}
$$

$L=0$.

For Maxwell's electromagnetic equations $\left(\Psi=\phi_{0}\right)$, the constant terms are

$$
\begin{aligned}
L_{3} & =-\frac{\partial g^{11} / \partial r-2\left(\partial g^{1 j} / \partial r\right) r_{h, j}+g^{j k} r_{h, j k}}{\left(g^{01}-g^{0 j} r_{h, j}\right)} \\
& +\frac{g^{j k} r_{h, j} r_{h, k}}{r_{h}^{2}\left(g^{01}-g^{0 j} r_{h, j}\right)}\left(r_{h}-1\right), \\
L_{4} & =\frac{-i}{\left(g^{01}-g^{0 j} r_{h, j}\right)}\left[\ell^{\nu}\left(n_{, v}^{1}-n_{, v}^{j} r_{h, j}\right)-m^{\nu}\left(\ell_{, v}^{1}\right.\right. \\
& \left.-\ell_{, \nu}^{j} r_{h, j}\right)-2 \rho\left(n^{1}-n^{j} r_{h, j}\right)+(\mu-2 \gamma)\left(\ell_{, v}^{1}\right. \\
& \left.-\ell_{, v}^{j} r_{h, j}\right)-(\pi-2 \alpha)\left(m^{1}-m^{j} r_{h, j}\right)+2 \tau\left(\bar{m}^{1}\right. \\
& \left.-\bar{m}^{j} r_{h, j}\right)+\frac{\left(\bar{m}^{1}-\bar{m}^{j} r_{h, j}\right)}{\left(\ell^{1}-\ell^{j} r_{h, j}\right)}\left\{m^{\nu}\left(\ell_{, v}^{1}-\ell_{, v}^{j} r_{h, j}\right)\right. \\
& -\ell^{\nu}\left(\bar{m}_{, v}^{1}-\bar{m}^{j} r_{h, j}\right) \\
& -\left\{\sigma\left(\bar{m}^{1}-\bar{m}^{j} r_{h, j}\right)-\kappa\left(n^{1}-n^{j} r_{h, j}\right)\right\} \\
& \left.\cdot \frac{\left(n^{1}-n^{j} r_{h, j}\right)\left(\bar{m}^{1}-\bar{m}^{j} r_{h, j}\right)}{\left(m^{1}-m^{j} r_{h, j}\right)\left(\ell^{1}-\ell^{j} r_{h, j}\right)}\right] .
\end{aligned}
$$


For $\left(\Psi=\phi_{1}\right)$

$$
\begin{aligned}
L_{3} & =-\frac{\partial g^{11} / \partial r-2\left(\partial g^{1 j} / \partial r\right) r_{h, j}+g^{j k} r_{h, j k}}{\left(g^{01}-g^{0 j} r_{h, j}\right)} \\
& +\frac{g^{j k} r_{h, j} r_{h, k}}{r_{h}^{2}\left(g^{01}-g^{0 j} r_{h, j}\right)}\left(r_{h}-1\right), \\
L_{4} & =\frac{-i}{\left(g^{01}-g^{0 j} r_{h, j}\right)}\left[-2 \rho\left(n^{1}-n^{j} r_{h, j}\right)+(\mu-2 \gamma)\right. \\
& \cdot\left(\ell^{1}-\ell^{j} r_{h, j}\right)+2 \tau\left(\bar{m}^{1}-\bar{m}^{j} r_{h, j}\right)-(\pi-2 \alpha)\left(m^{1}\right. \\
& \left.-m^{j} r_{h, j}\right)+n^{\nu}\left(\ell_{, v}^{1}-\ell_{, v}^{j} r_{h, j}\right)-\bar{m}^{v}\left(m_{, v}^{1}-m_{, v}^{j} r_{h, j}\right) \\
& +\frac{\left(\ell^{1}-\ell^{j} r_{h, j}\right)}{\left(\bar{m}^{1}-\bar{m}^{j} r_{h, j}\right)}\left\{\bar{m}^{v}\left(n_{, v}^{1}-n_{, v}^{j} r_{h, j}\right)\right. \\
& \left.-n^{\nu}\left(\bar{m}_{, v}^{1}-\bar{m}_{, v}^{j} r_{h, j}\right)\right\}+\left\{-\sigma\left(\bar{m}^{1}-\bar{m}^{j} r_{h, j}\right)\right. \\
& \left.\left.+\kappa\left(n^{1}-n^{j} r_{h, j}\right)\right\} \frac{\left(n^{1}-n^{j} r_{h, j}\right)}{\left(m^{1}-m^{j} r_{h, j}\right)}\right] .
\end{aligned}
$$

Lastly, for $\left(\Psi=\phi_{2}\right)$, we get

$$
\begin{aligned}
& L_{3}=-\frac{\partial g^{11} / \partial r-2\left(\partial g^{1 j} / \partial r\right) r_{h, j}+g^{j k} r_{h, j k}}{\left(g^{01}-g^{0 j} r_{h, j}\right)} \\
&+\frac{g^{j k} r_{h, j} r_{h, k}}{r_{h}^{2}\left(g^{01}-g^{0 j} r_{h, j}\right)}\left(r_{h}-1\right), \\
& L_{4}=\frac{-i}{\left(g^{01}-g^{0 j} r_{h, j}\right)}\left[n^{\nu}\left(\ell_{, v}^{1}-\ell_{, v}^{j} r_{h, j}\right)-\bar{m}^{v}\left(m_{, v}^{1}\right.\right. \\
&\left.-m_{, v}^{j} r_{h, j}\right)-2 \pi\left(m_{, v}^{1}-m_{, v}^{j} r_{h, j}\right)+(\tau-2 \beta)\left(\bar{m}_{, v}^{1}\right. \\
&\left.-\bar{m}^{j} r_{h, j}\right)-2(\rho-2 \epsilon)\left(n_{, v}^{1}-n_{, v}^{j} r_{h, j}\right)+2 \mu\left(\ell_{, v}^{1}\right. \\
&\left.-\ell_{, v}^{j} r_{h, j}\right)+\frac{\left(\ell^{1}-\ell^{j} r_{h, j}\right)}{\left(\bar{m}^{1}-\bar{m}^{j} r_{h, j}\right)} \\
&+\frac{\left(m^{1}-m^{j} r_{h, j}\right)}{\left(n^{1}-n^{j} r_{h, j}\right)}\left\{\lambda\left(n_{, v}^{1}-n_{, v}^{j} r_{h, j}\right)\right. \\
&\left.\left.-\bar{m}^{v}\left(n_{, v}^{1}-n_{, v}^{j} r_{h, j}\right)\right\} \frac{\left(m^{1}-m^{j} r_{h, j}\right)}{\left(n^{1}-n^{j} r_{h, j}\right)}\right] . \\
&\left.\left.{ }_{v} r_{h, j}\right)\right\}-2\left\{n^{v}\left(\bar{m}^{1}-\bar{m}^{j} r_{h, j}\right)\right.
\end{aligned}
$$

Similarly for Dirac particles when $\left(\Psi=f_{1}\right)$, (37) gives the following constant terms:

$$
\begin{aligned}
L_{3} & =-\frac{\partial g^{11} / \partial r-2\left(\partial g^{1 j} / \partial r\right) r_{h, j}+g^{j k} r_{h, j k}}{\left(g^{01}-g^{0 j} r_{h, j}\right)} \\
& +\frac{g^{j k} r_{h, j} r_{h, k}}{r_{h}^{2}\left(g^{01}-g^{0 j} r_{h, j}\right)}\left(r_{h}-1\right), \\
L_{4} & =\frac{-i}{\left(g^{01}-g^{0 j} r_{h, j}\right)}[-(\beta-\tau+\bar{\beta}-\bar{\tau}) \\
& \cdot\left(\bar{m}^{1}-\bar{m}^{j} r_{h, j}\right)+(\epsilon-\rho)\left(n^{1}-n^{j} r_{h, j}\right)+(\bar{\mu}-\bar{\gamma}) \\
& \cdot\left(\ell^{1}-\ell^{j} r_{h, j}\right) \\
& -\frac{\left(\ell^{1}-\ell^{j} r_{h, j}\right)}{\left(\bar{m}^{1}-\bar{m}^{j} r_{h, j}\right)}\left\{\bar{m}^{v}\left(n_{, v}^{1}-n_{, v}^{j}\right)+n^{\nu}\left(\bar{m}^{1}-\bar{m}_{, v}^{j}\right)\right\} \\
& -\left(\bar{\mu}^{-}-\bar{\gamma}^{\mu}+\gamma\right)\left(\ell^{1}-\ell^{j} r_{h, j}\right)-(\pi-\alpha-\bar{\beta}+\bar{\tau}) \\
& \left.\cdot \frac{\left(\ell^{1}-\ell^{j} r_{h, j}\right)^{2}}{\left(\bar{m}^{1}-\bar{m}^{j} r_{h, j}\right)}\right],
\end{aligned}
$$

and, for $\left(\Psi=f_{2}\right)$, we obtain

$$
\begin{aligned}
L_{3} & =-\left[\frac{\partial g^{11} / \partial r-2\left(\partial g^{1 j} / \partial r\right) r_{h, j}+g^{j k} r_{h, j k}}{\left(g^{01}-g^{0 j} r_{h, j}\right)}\right] \\
& +\frac{g^{j k} r_{h, j} r_{h, k}}{r_{h}^{2}\left(g^{01}-g^{0 j} r_{h, j}\right)}\left(r_{h}-1\right), \\
L_{4} & =\frac{-i}{\left(g^{01}-g^{0 j} r_{h, j}\right)}\left[(\mu-\gamma)\left(\ell^{1}-\ell^{j} r_{h, j}\right)-(\pi-\alpha)\right. \\
& \cdot\left(m^{1}-m^{j} r_{h, j}\right)+\ell^{v}\left(n_{, v}^{1}-n_{, v}^{j}\right)+(\bar{\epsilon}-\bar{\rho})\left(n^{1}\right. \\
& \left.-n^{j} r_{h, j}\right)+\ell^{v}\left(n_{, v}^{1}-n_{, v}^{j}\right)-m^{\nu}\left(\bar{m}^{1}-\bar{m}_{, v}^{j}\right)-(\bar{\epsilon}-\bar{\rho} \\
& -\epsilon+\rho)\left(n_{, v}^{1}-n_{, v}^{j}\right)-\ell^{\nu} \frac{\left(m_{, v}^{1}-m_{, v}^{j}\right)}{\left(\bar{m}^{1}-\bar{m}_{h, j}^{j}\right)}\left(n^{1}-n^{j} r_{h, j}\right) \\
& \left.\left.-(\beta-\tau-\bar{\pi}+\bar{\alpha}) \frac{\left(\ell^{1}-\ell^{j} r_{h, j}\right)}{\left(m^{1}-m^{j} r_{h, j}\right)}\right\}\left(n^{1}-n^{j} r_{h, j}\right)\right] . \\
& +\left\{m^{v} \frac{\left(\ell_{, v}^{1}-\ell_{, v}^{j}\right)}{\left(m^{1}-m^{j} r_{h, j}\right)}\right.
\end{aligned}
$$

Equation (37) may be assumed as second-order partial differential equation near the event horizon in general nonstationary black hole since all the coefficients $L_{1}, L_{2}, L_{3}$, and $L_{4}$ are constant terms when $r \rightarrow r_{h}\left(u_{0}, \theta_{0}, \phi_{0}\right), u \rightarrow u_{0}$, $\theta \rightarrow \theta_{0}$, and $\phi \rightarrow \phi_{0}$. 
Using [29, 30, 36, 52], the variables in (37) may be separated for the analysis of the field equations as

$$
\Psi=R\left(r_{*}, u_{*}\right) \Theta\left(u_{*}, \theta_{*}, \phi_{*}\right) e^{i \omega u_{*}+i K_{\theta}+i K_{\phi}},
$$

where $\Theta\left(u_{*}, \theta_{*}, \phi_{*}\right)$ is an arbitrary real function and $\omega$ is the energy of the particles which depend on tortoise coordinate transformation; $K_{\theta}$ and $K_{\phi}$ are components of generalized momentum of scalar particles. And we use $K_{\theta}=\partial S / \partial \theta_{*}$ and $K_{\phi}=\partial S / \partial \phi_{*}$, where $S$ is Hamiltonian function of scalar particles. Using (45) into (37) and after separating the variables, the radial and angular parts are given by

$$
\begin{aligned}
& \frac{\partial^{2} P}{\partial r_{*}^{2}}-\left(2 i \omega-L_{1} i K_{\theta}-L_{2} i K_{\phi}-L_{3}-i 2 L_{4}-\alpha\right) \frac{\partial P}{\partial r_{*}} \\
& \quad=0, \\
& \frac{2 \partial T}{\partial u_{*}}-\left(\zeta\left(u_{*}\right)-\alpha\right) T=0,
\end{aligned}
$$

$$
\widetilde{\Psi}_{\omega}^{\text {out }} \sim e^{i \omega+i K_{\theta}+i K_{\phi}} e^{(\pi / \kappa)\left[\omega-K_{\theta} L_{1} / 2-K_{\phi} L_{2} / 2-L_{4}\right]} e^{(i \pi / 2 \kappa)\left(\alpha+L_{3}\right)}\left(\frac{r_{h}-r}{r_{h}}\right)^{(i / \kappa)\left(\omega-K_{\theta} L_{1} / 2-K_{\phi} L_{2} / 2-L_{4}\right)}\left(\frac{r_{h}-r}{r_{h}}\right)^{(-1 / 2 \kappa)\left(\alpha+L_{3}\right)}, \quad r<r_{h} .
$$

From (47) and (48), one can obtain the relative scattering probability near the event horizon $r=r_{h}$ :

$$
\left|\frac{\Psi_{\omega}^{\text {out }}\left(r>r_{h}\right)}{\widetilde{\Psi_{\omega}^{\text {out }}}\left(r<r_{h}\right)}\right|^{2}=e^{(-2 \pi / \kappa)\left(\omega-\omega_{0}\right)}
$$

where

$$
\omega_{0}=K_{\theta} \frac{L_{1}}{2}+K_{\phi} \frac{L_{2}}{2}+L_{4}
$$

Following Damour and Ruffini [27] and extended by Sannan [28], the thermal radiation spectrum of Maxwell's electromagnetic field equations (Dirac particles or scalar particles) from general nonstationary black holes is given by

$$
N_{\omega}=\frac{1}{e^{\left(\omega-\omega_{0}\right) / \kappa_{B} T} \pm 1}
$$

where $\kappa_{B}$ is Boltzmann constant and upper positive symbol stands for the Fermi-Dirac distribution and the lower negative symbol corresponds to the Bose-Einstein statistics, showing that black hole radiates like a black body radiation. The Hawking temperature is given by

$$
\begin{aligned}
T & \left(u_{0}, \theta_{0}, \phi_{0}\right) \\
& =\frac{1}{2 \pi}\left[\frac{\partial g^{11} / \partial r-2\left(\partial g^{1 j} / \partial r\right) r_{h, j}+\left(\partial g^{j k} / \partial r\right) r_{h, j} r_{h, k}}{2\left\{g^{01}-2 g^{11}+\left(2 g^{1 j}-g^{0 j}\right) r_{h, j}\right\}}\right. \\
& \left.+\frac{g^{j k} r_{h, j} r_{h, k}-g^{1 j} r_{h, j}}{r_{h}\left\{g^{01}-2 g^{11}+\left(2 g^{1 j}-g^{0 j}\right) r_{h, j}\right\}}\right],
\end{aligned}
$$

where $\alpha$ and $\zeta\left(u_{*}\right)$ are a constant and function of retarded time $u_{*}$ in variable of separation, respectively, where $\zeta\left(u_{*}\right)=$ $\left.\left.2\left(\left(\partial \Theta / \partial u_{*}\right) / \Theta\right)+C_{1}\left(\left(\partial \Theta / \partial \theta_{*}\right)\right) / \Theta\right)-C_{2}\left(\left(\partial \Theta / \partial \phi_{*}\right) / \Theta\right)\right)$ and $R\left(r_{*}, u_{*}\right)=P\left(r_{*}\right) T\left(u_{*}\right)$.

After separation of variables, the radial components of two independent solutions are defined by

$$
\begin{aligned}
\Psi_{\omega}^{\text {in }} & \sim e^{i \omega+i K_{\theta}+i K_{\phi}}, \\
\Psi_{\omega}^{\text {out }} & \sim e^{i \omega+i K_{\theta}+i K_{\phi}} e^{2 i\left(\omega-K_{\theta} L_{1} / 2-K_{\phi} L_{2} / 2-L_{4}\right) r_{*}} e^{-\left(\alpha+L_{3}\right) r_{*}}, \\
r & >r_{h},
\end{aligned}
$$

where $\Psi_{\omega}^{\text {in }}$ represents an incoming wave which is analytic at $r=r_{h} ; \Psi_{\omega}^{\text {out }}$ denotes an outgoing wave having singularity at the event horizon. References $[27,28]$ indicate that $\Psi_{\omega}^{\text {out }}$ can continue analytically from outside of the event horizon $r=r_{h}$ into inside by rotating $-\pi$ through lower half of the complex plane as and the chemical potential is given by

$$
\omega_{0}=K_{\theta} \frac{g^{12}-g^{2 j} r_{h, j}}{g^{01}-g^{0 j} r_{h, j}}+K_{\phi} \frac{g^{13}-g^{3 j} r_{h, j}}{g^{01}-g^{0 j} r_{h, j}}+L_{4}
$$

Integrating the thermal radiation spectra (51) or distribution function over all $\omega$ 's the combined form of Hawking flux for Klein-Gordon scalar particles, Maxwell's electromagnetic field equations, and Dirac equations can be obtained as follows:

$$
\operatorname{Flux}=\frac{1}{\pi} \int_{0}^{\infty} \frac{\omega d \omega}{e^{(2 \pi / \kappa)\left(\omega-\omega_{0}\right)} \pm 1}
$$

This is an exact result for the energy flux in general nonstationary black hole. If $\omega_{0}=0$ in (54), the Hawking flux for fermions is given by

$$
(\text { Flux })_{\mid \text {fermions }}=\frac{1}{\pi} \int_{0}^{\infty} \frac{\omega d \omega}{e^{2 \pi \omega / \kappa}+1}=\frac{\kappa^{2}}{48 \pi},
$$

and the Hawking flux for boson is defined by

$$
(\text { Flux })_{\mid \text {boson }}=\frac{1}{\pi} \int_{0}^{\infty} \frac{\omega d \omega}{e^{2 \pi \omega / \kappa}-1}=\frac{\kappa^{2}}{24 \pi} .
$$

These results are consistent with ones already obtained in the literature $[53,54]$. From (52), we observe that $T$ is a function of retarded time and different angles. Hence, it is a distribution of temperature of the thermal radiation near the event horizon $r=r_{h}$ due to the Klein-Gordon scalar field, the Maxwell's electromagnetic field equations, 
and the Dirac equations in general nonstationary black hole. It has been shown that the constant coefficient $L_{4}$ appears in the expression of chemical potential and may represent a particular energy term for Maxwell's electromagnetic field and Dirac particles which is absent in the thermal radiation spectrum of other scalar particles.

\section{Nonthermal Radiation}

The relativistic Hamilton-Jacobi equation for the classical action of a particle of mass $\mu_{0}$ in a curve space-time is given by $[55]$

$$
g^{a b}\left(\frac{\partial \Phi}{\partial x^{a}}\right)\left(\frac{\partial \Phi}{\partial x^{b}}\right)-\mu_{0}^{2}=0
$$

where $\Phi=\Phi(u, r, \theta, \phi)$ is the Hamiltonian principal function. Using (3) into (57), we obtain the following:

$$
\begin{aligned}
& \frac{G}{2 \kappa\left(r-r_{h}\right) r_{h}}\left(\frac{\partial S}{\partial r_{*}}\right)^{2}-2 D\left(\frac{\partial S}{\partial r_{*}}\right)+2 r_{h} \kappa\left(r-r_{h}\right) Y \\
& =0
\end{aligned}
$$

where

$$
\begin{aligned}
G= & g^{11} r_{h}^{2}\left[1+2 \kappa\left(r-r_{h}\right)\right]^{2}+r^{2} g^{j k} r_{h, j} r_{h, k} \\
& -2 r r_{h}\left[1+2 \kappa\left(r-r_{h}\right)\right] r_{h, j} g^{1 j}, \\
D= & r g^{j k}\left(\frac{\partial S}{\partial x_{*}^{j}}\right)-r_{h}\left(\frac{\partial S}{\partial x_{*}^{j}}\right)\left[1+2 \kappa\left(r-r_{h}\right)\right], \\
Y= & g^{j k}\left(\frac{\partial S}{\partial x_{*}^{j}}\right)\left(\frac{\partial S}{\partial x_{*}^{k}}\right)+\mu_{0}^{2} .
\end{aligned}
$$

Multiplying by the factor $1 /\left[r_{h} g^{01}\left\{1+2 \kappa\left(r-r_{h}\right\}-r g^{0 j} r_{h, j}\right]\right.$ to both sides of (58) and assuming the resulting coefficient of $\left(\partial S / \partial r_{*}\right)^{2}$ tends to unity near the event horizon, then we get

$$
\begin{aligned}
& \lim _{r \rightarrow r_{h}} \frac{g^{11} r_{h}^{2}\left\{1+2 \kappa\left(r-r_{h}\right)\right\}^{2}-2 g^{1 j} r r_{h} r_{h, \phi}\left\{1+2 \kappa\left(r-r_{h}\right)\right\}}{2 \kappa r_{h}\left(r-r_{h}\right)\left[r_{h} g^{01}\left\{1+2 \kappa\left(r-r_{h}\right)\right\}-r g^{0 j} r_{h, j}\right]} \\
& \quad=1 .
\end{aligned}
$$

Equation (60) is an indeterminate form of $0 / 0$ near the black hole event horizon and, using L'Hospital's rule and adjusting parameter $\kappa$, the surface gravity obtained from relativistic Hamilton-Jacobi equation is given by

$$
\begin{aligned}
\kappa= & \frac{\partial g^{11} / \partial r-2\left(\partial g^{1 j} / \partial r\right) r_{h, j}+\left(\partial g^{j k} / \partial r\right) r_{h, j} r_{h, k}}{2\left[g^{01}-2 g^{11}+\left(2 g^{1 j}-g^{0 j}\right) r_{h, j}\right]} \\
& +\frac{g^{j k} r_{h, j} r_{h, k}-g^{1 j} r_{h, j}}{r_{h}\left[g^{01}-2 g^{11}+\left(2 g^{1 j}-g^{0 j}\right) r_{h, j}\right]}
\end{aligned}
$$

which is equal to the surface gravity obtained from KleinGordon equation, Maxwell's equations, and Dirac equations as given in (10), (26), and (36). Using the generalized tortoise coordinate transformation in (57) and defining $\partial \Phi / \partial u_{*}=$ $-\omega, \partial \Phi / \partial \theta_{*}=K_{\theta}$, and $\partial \Phi / \partial \phi_{*}=K_{\phi}$, for real $\partial \Phi / \partial r_{*}$, the distribution of energy levels of the particles is given by

$$
\begin{aligned}
& \omega \geq \omega^{+}, \\
& \omega \leq \omega^{-} .
\end{aligned}
$$

Near the black hole event horizon there exist seas of positiveand negative-energy states and a forbidden energy gap. Penrose [56] proposed that a particle entering close to the surface of the event horizon decays into two particles: one particle having positive energy escapes to infinity and the other particle having negative energy gets absorbed by the black hole. A quantum analogue as spontaneous pair creation was proposed by Zel'dovich [57] in the Kerr black hole. The energy states must satisfy the condition $\omega^{-} \leq \omega \leq \omega^{+}$at the forbidden region. The maximum value of the negative-energy state after overlapping of the positive- and negative-energy states at the surface of the event horizon is

$$
\omega_{h}=K_{\theta} \frac{g^{12}-g^{2 j} r_{h, j}}{g^{01}-g^{0 j} r_{h, j}}+K_{\phi} \frac{g^{13}-g^{3 j} r_{h, j}}{g^{01}-g^{0 j} r_{h, j}} .
$$

The width of the forbidden energy approaches zero near the event horizon. This indicates that there exists a crossing of the positive- and the negative-energy levels near the event horizon [38]. When $\omega_{h}>\mu_{0}$, the particle can escape to infinity from the black hole event horizon. The Starobinski-Unruh process (spontaneous radiation) must occur in the region near the black hole event horizon [57-61]. It indicates that the incident negative-energy particle will become emerging positive-energy particle via quantum tunneling effect. From this result, there is radiation near the event horizon. This type of radiation is independent of the temperature of the black hole and the type of quantum effect is a nonthermal.

Application of This Theory. The line element describing general nonstationary symmetrical black hole in retarded time coordinates $(u, r, \theta, \phi)$ is defined by

$$
\begin{aligned}
d s^{2}= & g_{00} d u^{2}+2 g_{01} d u d r+2 g_{02} d u d \theta+g_{03} d u d \phi \\
& +g_{13} d r d \phi+g_{22} d \theta^{2}+2 g_{23} d \theta d \phi+g_{33} d \phi^{2}
\end{aligned}
$$

Using (4), (5), and (64), the expression of temperature in general nonstationary axial symmetrical black hole is 


$$
T=\frac{1}{4 \pi r_{h}}\left[\frac{r_{h}\left(\partial g^{11} / \partial r-2\left(\partial g^{1 j} / \partial r\right) r_{h, j}+\left(\partial g^{j k} / \partial r\right) r_{h, j} r_{h, k}\right)+2\left(g^{j k} r_{h, j} r_{h, k}-g^{1 j} r_{h, j}\right)}{\left\{g^{01}-2 g^{11}+\left(2 g^{1 j}-g^{0 j}\right) r_{h, j}\right\}}\right]
$$

and the expression of chemical potential is defined by

$$
\omega_{0}=K_{\theta} \frac{g^{12}-g^{2 j} r_{h, j}}{g^{01}-g^{0 j} r_{h, j}}+K_{\phi} \frac{g^{13}-g^{3 j} r_{h, j}}{g^{01}-g^{0 j} r_{h, j}} .
$$

From (63) and (66), we observe that the chemical potential derived from scalar particle is equal to the highest energy of the negative-energy state.

In particular, the line element of Kerr black hole in retarded time coordinate is given by [62]

$$
\begin{aligned}
d s^{2}= & \left(1-2 M r R^{-2}\right) d u^{2}+2 d u d r \\
& +4 a r M R^{-2} \sin ^{2} \theta d u d \phi-2 a \sin ^{2} \theta d r d \phi \\
& -R^{2} d \theta^{2}-\left\{\left(r^{2}+a^{2}\right)-\Delta a^{2} \sin ^{2} \theta\right\} R^{-2} \theta^{2} d \phi^{2},
\end{aligned}
$$

where $\Delta=r^{2}-2 M r+a^{2}$ and $R^{2}=r^{2}+a^{2} \cos ^{2} \theta$. The Kerr black hole has singularity at $\Delta=0$. The roots $r_{h}=M+\sqrt{M^{2}-a^{2}}$ and $r_{h}=M-\sqrt{M^{2}-a^{2}}$ represent external event horizon and internal Cauchy horizon, respectively. From (65) and (67), the Hawking temperature of nonstationary Kerr black hole is obtained as

$$
\begin{aligned}
T= & \frac{1}{2 \pi} \\
& \cdot \frac{r_{h}^{2}\left(1+2 r_{h, u}\right)-r_{h} M\left(u_{0}\right)-\left\{\Delta_{h}+r_{h, u}\left(r_{h}^{2}+a^{2}\right)+a r_{h, \phi}\right\}}{r_{h}\left\{\left(r_{h}^{2}+a^{2}+a^{2} \sin ^{2} \theta_{0}\right)\left(1+2 r_{h, u}\right)+Z\right\}},
\end{aligned}
$$

where $Z=2 r_{h, \theta}^{2}+r_{h, \phi}\left(4 a r_{h, u}+2 r_{h, \phi} / \sin ^{2} \theta_{0}+3 a\right)$ and the chemical potential is

$$
\begin{aligned}
\omega_{0}= & \frac{K_{\theta} r_{h, \theta}}{\left[\left(r_{h}^{2}+a^{2}\right)+a^{2} \sin ^{2} \theta_{0} r_{h, u}+a r_{h, \phi}\right]} \\
& +\frac{K_{\phi}\left(r_{h, u}+a^{2} \sin ^{2} \theta_{0}+r_{h, \phi}\right)}{\sin ^{2} \theta_{0}\left[\left(r_{h}^{2}+a^{2}\right)+a^{2} \sin ^{2} \theta_{0} r_{h, u}+a r_{h, \phi}\right]} .
\end{aligned}
$$

This indicates that the chemical potential derived from scalar particles is equal to highest energy of the negative-energy state. This was mentioned at the beginning and has been shown by direct calculation to a special case.

\section{Discussion}

The total interaction energy of scalar particles of KleinGordon, Maxwell's electromagnetic field equations, and Dirac particles in general nonstationary black hole is given by

$$
\omega_{0}=K_{\theta} \frac{g^{12}-g^{2 j} r_{h, j}}{g^{01}-g^{0 j} r_{h, j}}+K_{\phi} \frac{g^{13}-g^{3 j} r_{h, j}}{g^{01}-g^{0 j} r_{h, j}}+L_{4}
$$

where

$$
L_{4}=0 \text {, }
$$

for $\Psi=\Phi$ (Klein-Gordon scalar particle),

$$
\begin{aligned}
L_{4} & =\frac{-i}{\left(g^{01}-g^{0 j} r_{h, j}\right)}\left[\ell^{v}\left(n_{, v}^{1}-n_{, v}^{j} r_{h, j}\right)-m^{\nu}\left(\ell_{, v}^{1}\right.\right. \\
& \left.-\ell_{, v}^{j} r_{h, j}\right)-2 \rho\left(n^{1}-n^{j} r_{h, j}\right)+(\mu-2 \gamma)\left(\ell_{, v}^{1}\right. \\
& \left.-\ell_{, v}^{j} r_{h, j}\right)-(\pi-2 \alpha)\left(m^{1}-m^{j} r_{h, j}\right)+2 \tau\left(\bar{m}^{1}\right. \\
& \left.-\bar{m}^{j} r_{h, j}\right)+\frac{\left(\bar{m}^{1}-\bar{m}^{j} r_{h, j}\right)}{\left(\ell^{1}-\ell^{j} r_{h, j}\right)}\left\{m^{\nu}\left(\ell_{, v}^{1}-\ell_{, v}^{j} r_{h, j}\right)\right. \\
& \left.-\ell^{v}\left(\bar{m}_{, v}^{1}-\bar{m}^{j} r_{h, j}\right)\right\}-\left\{\sigma\left(\bar{m}^{1}-\bar{m}^{j} r_{h, j}\right)\right. \\
& \left.\left.-\kappa\left(n^{1}-n^{j} r_{h, j}\right)\right\} \frac{\left(n^{1}-n^{j} r_{h, j}\right)\left(\bar{m}^{1}-\bar{m}^{j} r_{h, j}\right)}{\left(m^{1}-m^{j} r_{h, j}\right)\left(\ell^{1}-\ell^{j} r_{h, j}\right)}\right],
\end{aligned}
$$

for $\Psi=\phi_{0}$ (Maxwell's electromagnetic field),

$$
\begin{aligned}
L_{4} & =D_{4}=\frac{-i}{\left(g^{01}-g^{0 j} r_{h, j}\right)}\left[-2 \rho\left(n^{1}-n^{j} r_{h, j}\right)+(\mu\right. \\
& -2 \gamma)\left(\ell^{1}-\ell^{j} r_{h, j}\right)+2 \tau\left(\bar{m}^{1}-\bar{m}^{j} r_{h, j}\right)-(\pi-2 \alpha) \\
& \cdot\left(m^{1}-m^{j} r_{h, j}\right)+n^{\nu}\left(\ell_{, \nu}^{1}-\ell_{, \nu}^{j} r_{h, j}\right)-\bar{m}^{v}\left(m_{, v}^{1}\right. \\
& \left.-m_{v}^{j} r_{h, j}\right)+\frac{\left(\ell^{1}-\ell^{j} r_{h, j}\right)}{\left(\bar{m}^{1}-\bar{m}^{j} r_{h, j}\right)}\left\{\bar{m}^{v}\left(n_{, v}^{1}-n_{, \nu}^{j} r_{h, j}\right)\right. \\
& \left.-n^{\nu}\left(\bar{m}_{, \nu}^{1}-\bar{m}_{, v}^{j} r_{h, j}\right)\right\}+\left\{-\sigma\left(\bar{m}^{1}-\bar{m}^{j} r_{h, j}\right)\right. \\
& \left.\left.+\kappa\left(n^{1}-n^{j} r_{h, j}\right)\right\} \frac{\left(n^{1}-n^{j} r_{h, j}\right)}{\left(m^{1}-m^{j} r_{h, j}\right)}\right],
\end{aligned}
$$

for $\Psi=\phi_{1}$, and

$$
\begin{aligned}
L_{4} & =D_{4}=\frac{-i}{\left(g^{01}-g^{0 j} r_{h, j}\right)}\left[n^{\nu}\left(\ell_{, v}^{1}-\ell_{, v}^{j} r_{h, j}\right)\right. \\
& -\bar{m}^{v}\left(m_{, v}^{1}-m_{, v}^{j} r_{h, j}\right)-2 \pi\left(m_{, v}^{1}-m_{, v}^{j} r_{h, j}\right)+(\tau \\
& -2 \beta)\left(\bar{m}_{, v}^{1}-2(\rho-2 \epsilon)\left(n_{, \nu}^{1}-n_{, v}^{j} r_{h, j}\right)+2 \mu\left(\ell_{, \nu}^{1}\right.\right.
\end{aligned}
$$




$$
\begin{aligned}
& \left.-\ell_{, v}^{j} r_{h, j}\right)+\frac{\left(\ell^{1}-\ell^{j} r_{h, j}\right)}{\left(\bar{m}^{1}-\bar{m}^{j} r_{h, j}\right)} \\
& \cdot \frac{\left(m^{1}-m^{j} r_{h, j}\right)}{\left(n^{1}-n^{j} r_{h, j}\right)}\left\{\lambda\left(n_{, v}^{1}-n_{, v}^{j} r_{h, j}\right)\right. \\
& \left.-v\left(\bar{m}_{, v}^{1}-\bar{m}_{, v}^{j} r_{h, j}\right)\right\}-2\left\{n^{v}\left(\bar{m}^{1}-\bar{m}^{j} r_{h, j}\right)\right. \\
& \left.\left.-\bar{m}^{v}\left(n_{, v}^{1}-n_{, v}^{j} r_{h, j}\right)\right\} \frac{\left(m^{1}-m^{j} r_{h, j}\right)}{\left(n^{1}-n^{j} r_{h, j}\right)}\right]
\end{aligned}
$$

for $\Psi=\phi_{2}$.

$$
\begin{aligned}
L_{4} & =\frac{-i}{\left(g^{01}-g^{0 j} r_{h, j}\right)}\left[\ell^{v}\left(n_{, v}^{1}-n_{, v}^{j} r_{h, j}\right)-m^{\nu}\left(\ell_{, v}^{1}\right.\right. \\
& \left.-\ell_{, v}^{j} r_{h, j}\right)-2 \rho\left(n^{1}-n^{j} r_{h, j}\right)+(\mu-2 \gamma)\left(\ell_{, v}^{1}\right. \\
& \left.-\ell_{, v}^{j} r_{h, j}\right)-(\pi-2 \alpha)\left(m^{1}-m^{j} r_{h, j}\right)+2 \tau\left(\bar{m}^{1}\right. \\
& \left.-\bar{m}^{j} r_{h, j}\right)+\frac{\left(\bar{m}^{1}-\bar{m}^{j} r_{h, j}\right)}{\left(\ell^{1}-\ell^{j} r_{h, j}\right)}\left\{m^{v}\left(\ell_{, v}^{1}-\ell_{, v}^{j} r_{h, j}\right)\right. \\
& \left.-\ell^{v}\left(\bar{m}_{, v}^{1}-\bar{m}^{j} r_{h, j}\right)\right\}-\left\{\sigma\left(\bar{m}^{1}-\bar{m}^{j} r_{h, j}\right)\right. \\
& \left.\left.-\kappa\left(n^{1}-n^{j} r_{h, j}\right)\right\} \frac{\left(n^{1}-n^{j} r_{h, j}\right)\left(\bar{m}^{1}-\bar{m}^{j} r_{h, j}\right)}{\left(m^{1}-m^{j} r_{h, j}\right)\left(\ell^{1}-\ell^{j} r_{h, j}\right)}\right]
\end{aligned}
$$

for $\Psi=f_{1}$ (Dirac Particles) and

$$
\begin{aligned}
L_{4} & =\frac{-i}{\left(g^{01}-g^{0 j} r_{h, j}\right)}\left[(\mu-\gamma)\left(\ell^{1}-\ell^{j} r_{h, j}\right)-(\pi-\alpha)\right. \\
& \cdot\left(m^{1}-m^{j} r_{h, j}\right)+\ell^{v}\left(n_{, v}^{1}-n_{, v}^{j}\right)+(\bar{\epsilon}-\bar{\rho})\left(n^{1}\right. \\
& \left.-n^{j} r_{h, j}\right)+\ell^{v}\left(n_{, v}^{1}-n_{, v}^{j}\right)-m^{v}\left(\bar{m}^{1}-\bar{m}_{, v}^{j}\right)-(\bar{\epsilon}-\bar{\rho} \\
& -\epsilon+\rho)\left(n_{, v}^{1}-n_{, v}^{j}\right)-\ell^{v} \frac{\left(m_{, v}^{1}-m_{, v}^{j}\right)}{\left(\bar{m}^{1}-\bar{m}_{h, j}^{j}\right)}\left(n^{1}-n^{j} r_{h, j}\right) \\
& +\left\{m^{v} \frac{\left(\ell_{, v}^{1}-\ell_{, v}^{j}\right)}{\left(m^{1}-m^{j} r_{h, j}\right)}\right. \\
& \left.\left.-(\beta-\tau-\bar{\pi}+\bar{\alpha}) \frac{\left(\ell^{1}-\ell^{j} r_{h, j}\right)}{\left(m^{1}-m^{j} r_{h, j}\right)}\right\}\left(n^{1}-n^{j} r_{h, j}\right)\right]
\end{aligned}
$$

for $\Psi=f_{2}$.

This chemical potential $\omega_{0}$ is composed of two parts. The sum of the first two terms, that is, $2 K_{\theta}\left(\left(g^{12}-g^{2 j} r_{h, j}\right) /\left(g^{01}-\right.\right.$ $\left.\left.g^{0 j} r_{h, j}\right)\right)+2 K_{\phi}\left(\left(g^{13}-g^{3 j} r_{h, j}\right) /\left(g^{01}-g^{0 j} r_{h, j}\right)\right)$, is the rotational energy arising from the coupling between different components of generalized momentum of Maxwell's electromagnetic field or Dirac particles and different rotations of black hole. The second-term $L_{4}$ gives a new extra spinrotation coupling and spin acceleration coupling effect. The physical origin of extra coupling effect comes from the interaction between intrinsic spin of particles and generalized momentum of evaporating black hole but it has no classical correspondence $[29,52]$. The value of $L_{4}$ will vanish for the stationary black hole and scalar particle. When $L_{4}=0,(63)$ and (70) show the chemical potential derived from the thermal radiation spectrum of Maxwell's electromagnetic field or Dirac particle is equal to highest energy of the negativeenergy state of scalar particles in general nonstationary black hole. This brings out a clear relationship between the two kinds of radiation processes of black holes.

On the other hand, the quantitative causal relation with no-loss-no-gain character would be satisfied by a lot of general physical processes $[63,64]$. Using the no-loss-nogain homeomorphic map transformation satisfying causal relation, [65] derived the exact strain tensor formulas in Weitzenbock manifold. Reference [66] investigated the cosmic quantum birth by studying the Wheeler-DeWitt equation which satisfies quantitative causal relation. In fact, some effect of change (cause) of some quantities in (2) must result in the relative changes (cause) in (2) so as to keep no-loss-no-gain in the right hand side of (2), that is, zero. Similarly (6), (13), (15)(17), (27)-(29), and (57) must satisfy the quantitative causal relation in the same way. Hence the findings in this paper are consistent.

References [29-31] proposed the tortoise coordinate transformation which is applicable to the black hole event horizon

$$
\begin{aligned}
& r_{*}=r+\frac{1}{2 \widehat{\kappa}\left(u_{0}, \theta_{0}, \phi_{0}\right)} \ln \left\{r-r_{h}(u, \theta, \phi)\right\}, \\
& u_{*}=u-u_{0}, \\
& \theta_{*}=\theta-\theta_{0}, \\
& \phi_{*}=\phi-\phi_{0},
\end{aligned}
$$

and it gives the surface gravity at the event horizon as

$$
\begin{aligned}
\widehat{\kappa}\left(u_{0}, \theta_{0}, \phi_{0}\right) & \\
= & \frac{\partial g^{11} / \partial r-2\left(\partial g^{1 j} / \partial r\right) r_{h, j}+\left(\partial g^{j k} / \partial r\right) r_{h, j} r_{h, k}}{2\left[g^{01}-2 g^{11}+\left(2 g^{1 j}-g^{0 j}\right) r_{h, j}\right]},
\end{aligned}
$$

which is different from one given by (10), (26), and (36) corresponding to the generalized tortoise coordinate transformation (3). Using the different tortoise coordinate transformations (3) and (77), it has been observed that the surface gravities of the general nonstationary black hole for KleinGordon scalar particles, Maxwell's electromagnetic field equations, Dirac equations, and relativistic Hamilton-Jacobi equation near the black hole event horizon are the same as shown in (10), (26), (36), and (61) under transformation (3) but they are different for different tortoise coordinate 
transformations. From (61) and (78), the surface gravity can be written as

$$
\kappa\left(u_{0}, \theta_{0}, \phi_{0}\right)=\widehat{\kappa}\left(u_{0}, \theta_{0}, \phi_{0}\right)+\xi\left(u_{0}, \theta_{0}, \phi_{0}\right),
$$

where $\kappa\left(u_{0}, \theta_{0}, \phi_{0}\right)$ and $\widehat{\kappa}\left(u_{0}, \theta_{0}, \phi_{0}\right)$ are the surface gravities obtained from tortoise coordinate transformations (3) and (77). Similarly, (52) can be expressed as

$$
T\left(u_{0}, \theta_{0}, \phi_{0}\right)=\widehat{T}\left(u_{0}, \theta_{0}, \phi_{0}\right)+\frac{1}{2 \pi} \xi\left(u_{0}, \theta_{0}, \phi_{0}\right),
$$

where $\widehat{T}\left(u_{0}, \theta_{0}, \phi_{0}\right)$ is the thermal radiation temperature under tortoise coordinate transformation (77) and $\xi\left(u_{0}, \theta_{0}\right.$, $\phi_{0}$ ) denotes the difference of thermal radiation under the different tortoise coordinate transformations and it is given by

$$
\xi\left(u_{0}, \theta_{0}, \phi_{0}\right)=\frac{g^{j k} r_{h, j} r_{h, k}-g^{1 j} r_{h, j}}{r_{h}\left[g^{01}-2 g^{11}+\left(2 g^{1 j}-g^{0 j}\right) r_{h, j}\right]} .
$$

According to $[43,46]$, the correction rate under the different tortoise coordinate transformations is given by

$$
\begin{aligned}
& \Upsilon\left(u_{0}, \theta_{0}, \phi_{0}\right) \\
& =\frac{2\left(g^{j k} r_{h, j} r_{h, k}-g^{1 j} r_{h, j}\right)}{r_{h}\left(\partial g^{11} / \partial r-2\left(\partial g^{1 j} / \partial r\right) r_{h, j}+\left(\partial g^{j k} / \partial r\right) r_{h, j} r_{h, k}\right)} .
\end{aligned}
$$

If $\xi\left(u_{0}, \theta_{0}, \phi_{0}\right)$ approaches zero, the two surface gravities are equal due to the tortoise coordinate transformations (3) and (77). This indicates that different tortoise coordinate transformations correspond to different Hawking radiation temperatures in a nonstationary rotating black hole spacetime [45]. Equation (80) implies that when $\widehat{T}\left(u_{0}, \theta_{0}, \phi_{0}\right)$ become zero, the temperature $T\left(u_{0}, \theta_{0}, \phi_{0}\right)$ will not be vanished due to the presence of extra term $\xi\left(u_{0}, \theta_{0}, \phi_{0}\right)$. From this research paper, we conclude that the generalized tortoise coordinate transformation (3) provides an alternative and convenient way to obtain Hawking radiation and is also generalization and development of the works of $[36,52]$ about quantum radiation for general nonstationary black holes.

\section{Conclusions}

In this paper the thermal radiation and chemical potential are investigated using the generalized tortoise coordinate transformation in general nonstationary black hole. The locations of the horizon and the thermal radiation near the black hole event horizon are determined. It has been found that they are functions of retarded time coordinate $u$ and different angles $\theta, \phi$. By adjusting properly the value of $\kappa$, the secondorder form of Klein-Gordon equation, the three second-order forms of Maxwell's electromagnetic field equations, and the two second-order forms of Dirac equations are transformed into a standard form of wave equation near the event horizon $r=r_{h}$. Separating the variables of the wave equation and applying the well known Damour-Ruffini-Sannan method, we determine accurately the thermal radiation and chemical potential at the event horizon $r=r_{h}$ in general nonstationary black hole. It is also observed that the constant term $L_{4}$ appeared in the expression of chemical potential which gives the interaction between the intrinsic spin of the particles and the generalized momentum in general nonstationary black hole but it is found to be absent in the chemical potential derived from Klein-Gordon scalar particles. This paper reveals the chemical potential obtained from the thermal radiation spectrum of Klein-Gordon scalar particles is equal to the highest energy of the negative-energy states of the nonthermal radiation in general nonstationary black hole near the event horizon. Besides, under the generalized coordinate transformation a constant term $\xi\left(u_{0}, \theta_{0}, \phi_{0}\right)$ has appeared in the expression of surface gravity and the thermal radiation of black hole near the event horizon. As $\xi\left(u_{0}, \theta_{0}, \phi_{0}\right)$ tends to zero, our results are consistent with results already obtained by Hua and Huang [36, 52]. In conclusion, the generalized tortoise coordinate transformation is found to be more reliable and accurate in the study of thermal radiation spectrum in general nonstationary black hole near the event horizon $r=r_{h}$.

\section{Competing Interests}

The author declares that there is no conflict of interests regarding the publication of this paper.

\section{Acknowledgments}

The author would like to thank Professor Ng. Ibohal, Professor N. Nimai, and Dr. I. Ablu for illuminating discussions.

\section{References}

[1] S. W. Hawking, "Black hole explosions?" Nature, vol. 248, no. 5443, pp. 30-31, 1974.

[2] S. W. Hawking, "Particle creation by black holes," Communications in Mathematical Physics, vol. 43, no. 3, pp. 199-220, 1975.

[3] J. D. Bekenstein, "Black holes and entropy," Physical Review. D. Particles and Fields. Third Series, vol. 7, pp. 2333-2346, 1973.

[4] J. D. Bekenstein, "Generalized second law of thermodynamics in black-hole physics," Physical Review D, vol. 9, no. 12, pp. 3292$3300,1974$.

[5] J. M. Bardeen, B. Carter, and S. W. Hawking, "The four laws of black hole mechanics," Communications in Mathematical Physics, vol. 31, pp. 161-170, 1973.

[6] P. Kraus and F. Wilczek, "Self-interaction correction to black hole radiance," Nuclear Physics B, vol. 433, no. 2, pp. 403-420, 1995.

[7] M. K. Parikh and F. Wilczek, "Hawking radiation as tunneling," Physical Review Letters, vol. 85, no. 24, pp. 5042-5045, 2000.

[8] J. Zhang and Z. Zhao, "Massive particles' black hole tunneling and de Sitter tunneling," Nuclear Physics. B, vol. 725, no. 1-2, pp. 173-180, 2005.

[9] J. Zhang and Z. Zhao, "Charged particles' tunnelling from the Kerr-Newman black hole," Physics Letters. B, vol. 638, no. 2-3, pp. 110-113, 2006.

[10] J. Zhang and Z. Zhao, "Hawking radiation of charged particles via tunneling from the Reissner-Nordstorm black hole," Journal of High Energy Physics, vol. 2005, no. 10, 2005. 
[11] E. T. Akhmedov, V. Akhmedova, and D. Singleton, "Hawking temperature in the tunneling picture," Physics Letters. B, vol. 642, no. 1-2, pp. 124-128, 2006.

[12] E. T. Akhmedov, V. Akhmedova, D. Singleton, and T. Pilling, "Thermal radiation of various gravitational backgrounds," International Journal of Modern Physics A, vol. 22, no. 8-9, pp. 17051715, 2007.

[13] B. D. Chowdhury, "Problems with tunneling of thin shells from black holes," Pramana-Journal of Physics, vol. 70, no. 1, pp. 326, 2008.

[14] A. De Gill, D. Singleton, V. Akhmedova, and T. Pilling, "A WKBlike approach to Unruh radiation," American Journal of Physics, vol. 78, no. 7, pp. 685-691, 2010.

[15] V. Akhmedov, T. Pilling, A. de Gill, and D. Singleton, "Temporal contribution to gravitational WKB-like calculations," Physics Letters B, vol. 666, pp. 269-271, 2008.

[16] V. Akhmedova, T. Pilling, A. de Gill, and D. Singleton, "Comments on anomaly versus WKB/tunneling methods for calculating Unruh radiation,” Physics Letters. B, vol. 673, no. 3, pp. 227-231, 2009.

[17] E. T. Akhmedov, T. Pilling, and D. Singleton, "Subtleties in the quasi-classical calculation of Hawking radiation," International Journal of Modern Physics D, vol. 17, article 2453, 2008.

[18] R.-G. Cai, "A note on thermodynamics of black holes in Lovelock gravity," Physics Letters. B, vol. 582, no. 3-4, pp. 237242, 2004.

[19] R.-G. Cai, "Gauss-Bonnet black holes in AdS spaces," Physical Review D, vol. 65, no. 8, Article ID 08014, 9 pages, 2002.

[20] R.-G. Cai, "Cardy-Verlinde formula and thermodynamics of black holes in de Sitter spaces," Nuclear Physics. B, vol. 628, no. 1-2, pp. 375-386, 2002.

[21] M. Angheben, M. Nadalini, L. Vanzo, and S. Zerbini, "Hawking radiation as tunneling for extremal and rotating black holes," Journal of High Energy Physics, vol. 2005, no. 5, 2005.

[22] D. Chen and S. Yang, "Hamilton-Jacobi ansatz to study the Hawking radiation of Kerr-Newman-KASuya black holes," International Journal of Modern Physics A. Particles and Fields. Gravitation. Cosmology, vol. 22, no. 28, pp. 5173-5178, 2007.

[23] G. Wang, B. Liu, and W. Liu, "Coordinates problem of Hawking radiation derivation in a Kerr-Newman black hole using HAMilton-Jacobi equation," General Relativity and Gravitation, vol. 42, no. 3, pp. 633-640, 2010.

[24] D. Chen and S. Yang, "Charged particle tunnels from the stationary and non-stationary Kerr-Newman black holes," General Relativity and Gravitation, vol. 39, no. 9, pp. 1503-1515, 2007.

[25] T. I. Singh, I. A. Meitei, and K. Y. Singh, "Hawking radiation of Kerr-de Sitter black holes using Hamilton-Jacobi method," Astrophysics and Space Science, vol. 345, no. 1, pp. 177-181, 2013.

[26] R. Kerner and R. B. Mann, "Fermions tunnelling from black holes," Classical and Quantum Gravity, vol. 25, no. 9, Article ID 095014, 2008.

[27] T. Damour and R. Ruffini, "Black-hole evaporation in the KleinSauter-Heisenberg-Euler formalism," Physical Review D, vol. 14, no. 2, pp. 332-334, 1976.

[28] S. Sannan, "Heuristic derivation of the probability distributions of particles emitted by a black hole," General Relativity and Gravitation, vol. 20, no. 3, pp. 239-246, 1988.

[29] S. Q. Wu and X. Cai, "Hawking radiation of Dirac particles in a variable-mass Kerr space-time," General Relativity and Gravitation, vol. 33, no. 7, pp. 1181-1195, 2001.
[30] S. Q. Wu and X. Cai, "Hawking radiation of a non-stationary Kerr-Newman black hole: spin-rotation coupling effect," General Relativity and Gravitation, vol. 34, no. 5, pp. 605-617, 2002.

[31] N. Ibohal and T. Ibungochouba, "Hawking's radiation in nonstationary rotating de Sitter background," Astrophysics and Space Science, vol. 333, no. 1, pp. 175-185, 2011.

[32] W. A. Hiscock, "Models of evaporating black holes. II. Effects of the outgoing created radiation," Physical Review. D. Particles and Fields. Third Series, vol. 23, no. 12, pp. 2823-2827, 1981.

[33] R. Balbinot, "Back reaction and the small-mass regime," Physical Review D, vol. 33, no. 6, pp. 1611-1615, 1986.

[34] Y. Shuzheng and Z. Zheng, "Nonthermal radiation from nonstationary Kerr black hole," International Journal of Theoretical Physics, vol. 35, no. 12, pp. 2455-2460, 1996.

[35] J. Lu, "Quantum nonthermal radiation of the nonstationary Kerr-Newman black hole," International Journal of Theoretical Physics, vol. 38, no. 7, pp. 2029-2036, 1999.

[36] J.-C. Hua and Y.-C. Huang, "Quantum radiation of general nonstationary black holes," EPL, vol. 85, no. 3, Article ID 30007, 2009.

[37] T. I. Singh, "Hawking radiation of stationary and non-stationary Kerr-de Sitter black holes," Chinese Physics B, vol. 24, no. 7, Article ID 070401, 2015.

[38] M. H. Ali, "Quantum nonthermal radiation of nonstationary Kerr-Newman-de Sitter black holes," General Relativity and Gravitation, vol. 36, no. 5, pp. 1171-1181, 2004.

[39] Z. Zhao, The Thermal of Black Holes and the Singularity of the Spacetime, Beijing Normal University Press, Beijing, China, 1999.

[40] T. Ibungochouba Singh, I. Ablu Meitei, and K. Yugindro Singh, "Quantum radiation of Maxwell's electromagnetic field in nonstationary Kerr-de Sitter black hole," International Journal of Modern Physics D, vol. 25, no. 5, Article ID 1650061, 2016.

[41] I. A. Meitei, T. I. Singh, and K. Y. Singh, "Quantum nonthermal radiation of nonstationary rotating de Sitter cosmological model," International Journal of Modern Physics D, vol. 23, no. 9, Article ID 1450077, 10 pages, 2014.

[42] N. Ibohal and T. Ibungochouba, "New tortoise coordinate transformation and Hawking's radiation in de Sitter space," Astrophysics and Space Science, vol. 343, no. 1, pp. 471-479, 2013.

[43] T. I. Singh, "Hawking radiation of Kerr-Newman black hole in different tortoise coordinate transformations," Astrophysics and Space Science, vol. 347, no. 2, pp. 271-276, 2013.

[44] J. Yang, Z. Zhao, G.-H. Tian, and W.-B. Liu, “Tortoise coordinates and hawking radiation in a dynamical spherically symmetric spacetime," Chinese Physics Letters, vol. 26, no. 12, Article ID 120401, 2009.

[45] X.-G. Lan, "New tortoise coordinate and Hawking radiation of Dirac particles in a non-stationary Kerr-Newman black hole," International Journal of Theoretical Physics, vol. 51, no. 4, pp. 1195-1203, 2012.

[46] X. G. Lan, Q. Q. Jiang, and L. F. Wei, "Hawking radiation temperatures in non-stationary Kerr black holes with different tortoise coordinate transformations," European Physical Journal C, vol. 72, no. 4, article no. 1983, 2012.

[47] E. Newman and R. Penrose, "An approach to gravitational radiation by a method of spin coefficients," Journal of Mathematical Physics, vol. 3, pp. 566-578, 1962.

[48] M. Carmeli, Classical Fields: General Relativity and Gauge Theory, John Wiley \& Sons, New York, NY, USA, 1982. 
[49] S. Chandrasekhar, The Mathematical Theory of Black Holes, vol. 69 of International Series of Monographs on Physics, Oxford University Press, New York, NY, USA, 1983.

[50] W. B. Bonnor and P. C. Vaidya, "Spherically symmetric radiation of charge in Einstein-Maxwell theory," General Relativity and Gravitation, vol. 1, no. 2, pp. 127-130, 1970.

[51] D. N. Page, "Dirac equation around a charged, rotating black hole," Physical Review D, vol. 14, no. 6, pp. 1509-1510, 1976.

[52] J.-C. Hua and Y.-C. Huang, "Quantum radiation properties of Dirac particles in general nonstationary black holes," Advances in High Energy Physics, vol. 2014, Article ID 707519, 10 pages, 2014.

[53] C.-H. Wu and L. H. Ford, "Fluctuations of the Hawking flux," Physical Review D, vol. 60, no. 10, Article ID 104013, 1999.

[54] R. Banerjee and B. R. Majhi, "Hawking black body spectrum from tunneling mechanism," Physics Letters B, vol. 675, no. 2, pp. 243-245, 2009.

[55] T. Damour, "Introduction Lectures on the effective one body formalism," International Journal of Modern Physics A, vol. 23, no. 8, pp. 1130-1148, 2008.

[56] R. Penrose, "Gravitational collapse: the role of general relativity," Rivita del Cimento, Numero Speziale, vol. 1, p. 252, 1969.

[57] B. Y. Zel'dovich, "The Generation of Waves by a rotating body," Journal of Experimental and Theoritical Physics Letters, vol. 14, p. $180,1971$.

[58] A. A. Starobinsky, "Amplification of electromagnetic and gravitational waves scattered by a rotating black hole," Journal of Experimental and Theoritical Physics, vol. 37, pp. 28-32, 1973.

[59] W. G. Unruh, "Second quantization in the Kerr metric," Physical Review D, vol. 10, no. 10, pp. 3194-3205, 1974.

[60] A. L. Matacz, P. C. Davies, and A. C. Ottewill, "Quantum vacuum instability near rotating stars," Physical Review. D. Third Series, vol. 47, no. 4, pp. 1557-1562, 1993.

[61] P. C. Davies, "Quantum vacuum friction," Journal of Optics B. Quantum and Semiclassical Optics, vol. 7, no. 3, pp. S40-S46, 2005.

[62] N. Ibohal, "Rotating metrics admitting non-perfect fluids," General Relativity and Gravitation, vol. 37, no. 1, pp. 19-51, 2005.

[63] L. Liao and Y. C. Huang, "Non-equivalence of FADdeev-JACkiw method and Dirac-Bergmann algorithm and the modification of FADdeev-JACkiw method for keeping the equivalence," Annals of Physics, vol. 322, no. 10, pp. 2469-2484, 2007.

[64] Y.-C. Huang and C.-X. Yu, "Quantization and spectrum of an open 2-brane," Physical Review D, vol. 75, no. 4, Article ID 044011, 2007.

[65] Y. C. Huang and B. L. Lin, "General Weitzenbock theory of crystals with different dislocation distribution," Physics Letters A, vol. 299, no. 5-6, pp. 644-649, 2002.

[66] Y.-C. Huang and G. Weng, "Solution of Wheeler-De Witt equation, potential well and tunnel effect," Communications in Theoretical Physics, vol. 44, no. 4, pp. 757-761, 2005. 

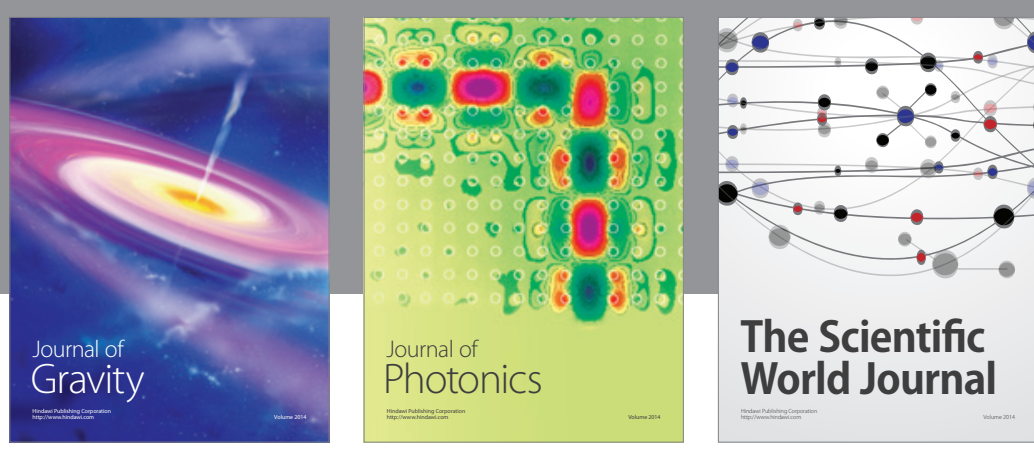

The Scientific World Journal
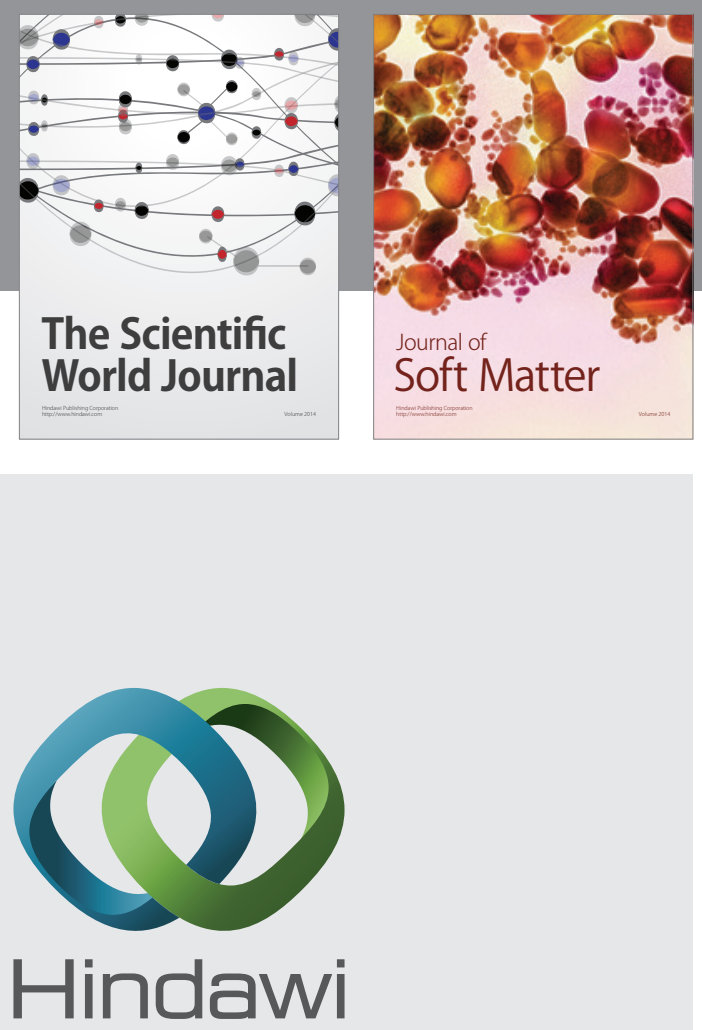

Submit your manuscripts at

https://www.hindawi.com
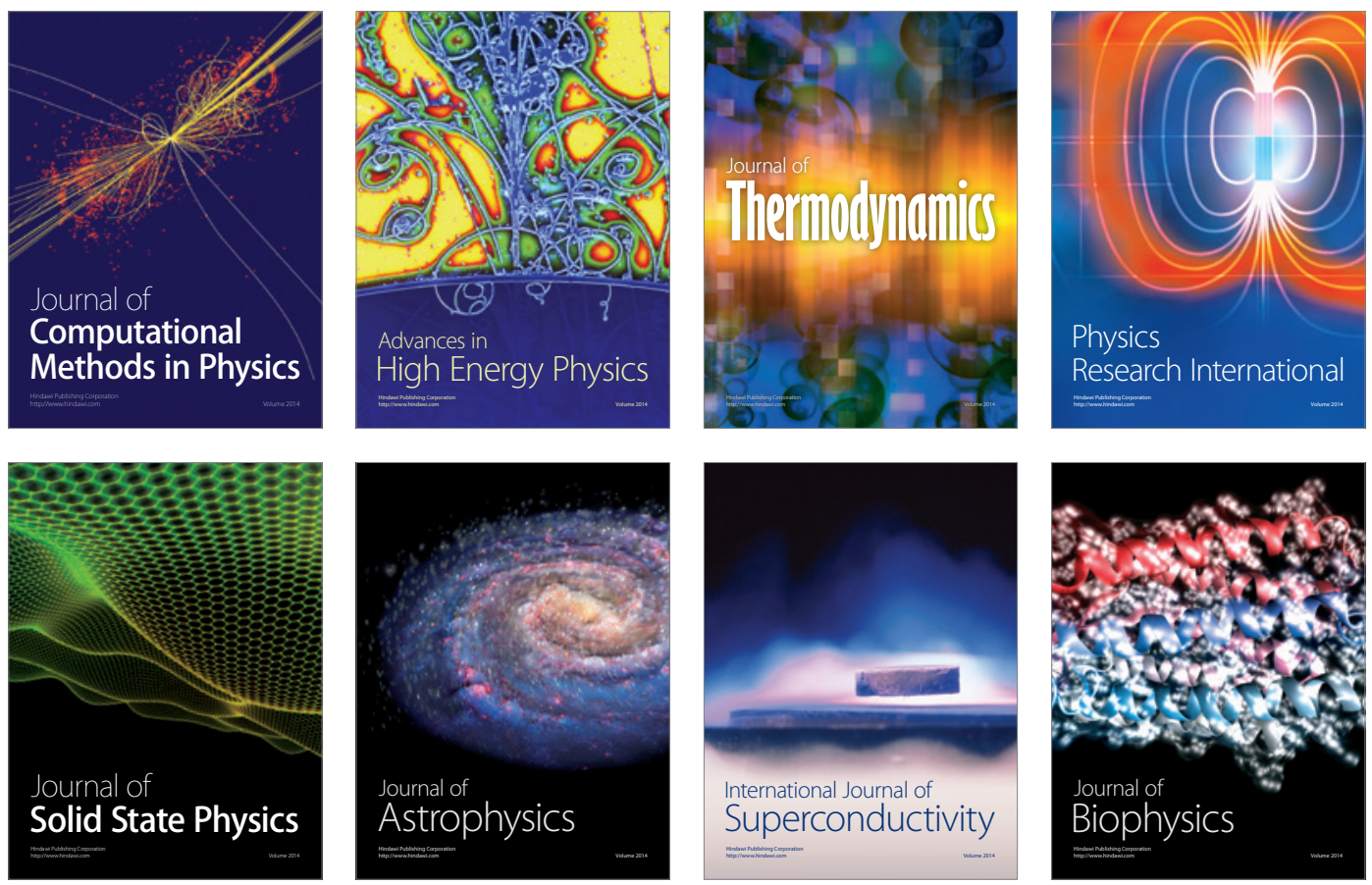
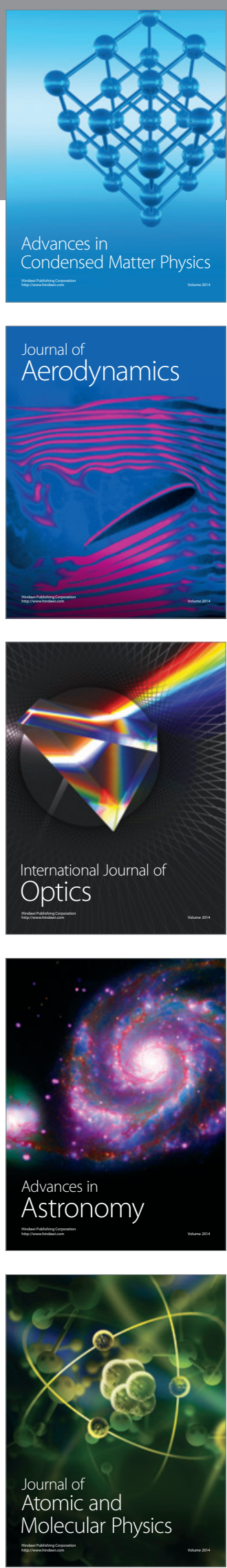